\title{
ON AN EXTENSION OF THE 1894 MEMOIR OF STIELTJES*
}

\author{
BY \\ EDWARD BURR VAN VLECK \\ INTRODUCTION
}

In the closing memoir $\dagger$ of his life STIELTJES gave an elegant and exhaustive discussion of the continued fraction

$$
\frac{1}{a_{1} z}+\frac{1}{a_{2}}+\frac{1}{a_{3} z}+\frac{1}{a_{4}}+\cdots,
$$

in which the elements $a_{n}$ are all positive. To this continued fraction he showed that there corresponds, on the one hand, a single series

$$
\frac{c_{0}}{z}-\frac{c_{1}}{z^{2}}+\frac{c_{3}}{z^{3}}-\frac{c_{4}}{z^{4}}+\cdots
$$

in which the coefficients are conditioned by the relations

$$
A_{n} \equiv\left|\begin{array}{cccc}
c_{0} & c_{1} & \cdots & c_{n-1} \\
c_{1} \cdot & c_{2} & \cdots & c_{n} \\
\cdot & \cdot & \cdot & \cdot \\
c_{n-1} & c_{n} & \cdots & c_{2 n-2}
\end{array}\right|>0, \quad B_{n} \equiv\left|\begin{array}{cccc}
c_{1} & c_{2} & \cdots & c_{n} \\
c_{2} & c_{3} & \cdots & c_{n+1} \\
\cdot & \cdot & \cdot & \cdot \\
c_{n} & c_{n+1} & \cdots & c_{2 n-1}
\end{array}\right|>0
$$

and, on the other hand, one or more functional integrals

$$
\int_{0}^{\infty} \frac{d \Phi(u)}{z+u}
$$

in which $\Phi(u)$ is a real and increasing function of $u$, though not necessarily analytic or continuous. Conversely, to every such series or integral (provided the integral permits of formal expansion into a series of the form (2)) there corre-

* Presented to the Society, February 28, 1903. Received for publication, May 25, 1903.

† Annales de la Faculté des Sciences de Toulouse, vol. 8 (1894), with continuation in vol. 9. The continued fraction of Stieltues is also treated in Blumenthal's Dissertation : Ueber die Entwicklung einer willkürlichen Funktion nach den Nennern des Kettenbruches für

Göttingen, 1898.

$$
\int_{-\infty}^{0} \frac{\Phi(\xi) d \xi}{z-\xi}
$$


sponds a single continued fraction of the form (1), and the elements of this continued fraction are positive.

In the extension of the work of STIELTJEs which I shall give here the amount of restriction imposed upon the continued fraction and series will be diminished by half. In the former it will be required either that $a_{2 n+1}>0$ or that $a_{2 n}>0$. The corresponding conditions for the series are that $A_{n}>0, B_{n} \neq 0$ or $B_{n}>0, A_{n} \neq 0$. If, furthermore, the even convergents in the one case and the odd convergents in the other are considered in separate continued fractions instead of being united by (1) into a single continued fraction, the subsidiary conditions $A_{n} \neq 0$ and $B_{n} \neq 0$ may be dispensed with entirely.

The integrals which correspond to the series and continued fractions, in case they exist, are of the form

$$
\int_{b}^{a} \frac{d \Phi(u)}{z+u} \quad(-\infty \leqq b<a \leqq+\infty),
$$

$\Phi(u)$ being of the same character as in (3). The change thus made in the limits of integration brings the work of STIELTJes into close connection with integrals considered by Heine, TschebyschefF, and others.

If the continued fraction (1) is thrown with the aid of the substitution $z=1 / x$ into the more familiar form

$$
\frac{b_{1} x}{1}+\frac{b_{2} x}{1}+\frac{b_{3} x}{1}+\cdots \quad\left(b_{n}=\frac{1}{a_{n} a_{n-1}}\right),
$$

the restrictions upon the coefficients are that $b_{2 n}$ and $b_{2 n+1}$ (or $b_{2 n+1}$ and $b_{2 n+2}$ ) shall always have a common sign, the sign, however, being arbitrary for each value of $n$.

The condition imposed by StIELTJES upon the coefficients of (1) seems to be the simplest one possible. Not only does it suffice to ensure the reality of the roots of the numerators and denominators of the convergents, but certain theorems* also follow concerning the alternation of the roots when two convergents are compared. On these theorems STIELTJES rears his structure. In Section I of the present paper I have shown that these theorems will also hold for the more general class of continued fractions here treated, if the positive and negative halves of the real axes are considered apart.

In most particulars the theory of these continued fractions is parallel to that of Stieltues, and his line of thought can be modified so as to apply. The greatest differences between the two theories appear in matters of convergence. Stieltues distinguishes two cases of his continued fraction. When $\sum_{n=1}^{\infty} a_{n}$ is divergent, the continued fraction converges over the entire plane of $z$ with the exception of a part or the whole of the negative half of the real axis, and its

* Loc. cit., \& 3-5. 
limit is an analytic function which can be expressed by means of an integral of the form (3). The corresponding series does not, however, necessarily converge. If, in short, $c_{n} / c_{n-1}$, which increases with $n$, increases beyond all limit, the series is divergent. A special interest attaches to this case, because the divergent series still determines uniquely a convergent continued fraction and therefore an analytic function.

When $\sum a_{n}$ is convergent, the continued fraction itself does not converge, but the even and odd convergents separately do, thereby defining two functions. STIELTJEs shows that the numerators and denominators of the two sets of convergents also have limits, and these limits are holomorphic functions of genre 0 . The corresponding series (2) is, of course, divergent.

For the generalized continued fractions before us the matter is far more complicated. No necessary and sufficient test for convergence has been found, and it seems quite probable that no such test is possible. Criteria are, however, given which will cover most of the cases likely to arise. Whenever the continued fraction converges uniformly in any region, the region of convergence covers the entire plane of $z$ with the exception of the whole or a part of the real axis, and the limit is holomorphic within this region. If the whole axis is to be excluded, it does not follow of necessity that the analytic functions determined in the two half planes are distinct, but it is interesting to note that such a case does actually occur. The infinite series which corresponds to the continued fraction is divergent, and we have therefore the remarkable phenomenon, so far as I know never previously pointed out, that a divergent power series may define two analytic functions separated by a natural boundary.

In addition to the two special cases of the continued fraction distinguished by StieltJes one other may be mentioned, in which the alternate even convergents form two separate sequences. In either sequence the limits of the numerator and denominator are holomorphic functions of genre 1 or 0 . The odd convergents, on the other hand, constitute a single sequence of the same character.

The last section of the paper is devoted to an examination of the table of rational approximants for the series (2), constructed after the manner of PADE. The table is "normal" for the continued fraction of STIELTJES and contains only irregularities of the first order when $A_{n}>0$ or $B_{n}>0$. The paper closes with a brief consideration of the zeros of the numerators and denominators of the approximants. The most interesting result here reached is published separately* and relates to the approximant obtained by taking the first $2 n+2$ or $2 n+1$ terms of $(2)$. This polynomial has the maximum number of imaginary roots, $2 n$, thus differing essentially from the approximants used as the convergents of $(1)$, the zeros and infinities of which are all real.

\footnotetext{
* Annals of Mathematics, July, 1903.
} 
No attempt is made to give a complete discussion of all the questions which arise in the extension of the memoir of STIELTJEs, but much is left for subsequent investigation.

\section{O, the Zeros and Infinities of the Convergents of the Continued Fraction.}

1. The formation of a Sturm series.-Let $N_{n}$ and $D_{n}$ denote the numerator and denominator of the $n$th convergent of a continued fraction

$$
\frac{1}{1}+\frac{b_{2} x}{1}+\frac{b_{3} x}{1}+\cdots
$$

in which the elements $b_{n}$ are real. From the fundamental relations

$$
\begin{aligned}
& D_{n}=D_{n-1}+b_{n} x D_{n-2}, \\
& N_{n}=N_{n-1}+b_{n} x N_{n-2},
\end{aligned}
$$

it can be shown at once by mathematical induction that $D_{n}$ and $N_{n}$ have for an even subscript the form

$$
\begin{aligned}
& D_{2 n}=1+\left(b_{2}+b_{3}+b_{4}+\cdots+b_{2 n}\right) x+\cdots+b_{2} b_{4} \cdots b_{2 n} x^{n}, \\
& N_{2 n}=1+\left(b_{3}+b_{4}+\cdots+b_{2 n}\right) x+\cdots+(\quad) x^{n-1},
\end{aligned}
$$

and for an odd subscript

$$
\begin{aligned}
& D_{2 n+1}=1+\left(b_{2}+b_{3}+\cdots+b_{2 n+1}\right) x+\cdots+(\quad) x^{n}, \\
& N_{2 n+1}=1+\left(b_{3}+b_{4}+\cdots+b_{2 n+1}\right) x+\cdots+b_{3} b_{5} \cdots b_{2 n+1} x^{n} .
\end{aligned}
$$

From (5) we obtain further

$$
D_{n}=\left(1+b_{n-1} x+b_{n} x\right) D_{n-2}-b_{n-1} b_{n-2} x^{2} D_{n-4}
$$

and a similar formula for $N_{n}$.

The last equation gives immediately a necessary condition that the alternate denominators (or numerators) of the convergents shall form a Sturm series. Since $D_{n}$ and $D_{n-4}$ must have opposite signs when $D_{n-2}$ vanishes, either $b_{2 n-1} b_{2 n}$ or $b_{2 n} b_{2 n+1}$ must be positive for all values of $n$. It is really immaterial which supposition we take. For should $b_{2 n}$ and $b_{2 n+1}$ have like signs, by dropping the first partial fraction of (4) we obtain another continued fraction in which the $(2 n-1)$ th and $2 n$th coefficients have a common sign. Now the denominators of the latter continued fraction are the numerators of the convergeuts of the former. The results of either supposition can therefore be derived 
readily from those of the other. As also the consequences of the two suppositions are in every way parallel, it will suffice to develop here only one of the two.

We will accordingly suppose that $b_{2 n}$ and $b_{2 n+1}$ are always of the same sign, the sign being arbitrary for each value of $n$. Consider then the sequence

$$
D_{0}=1, D_{2}, D_{4}, \cdots, D_{2 n} .
$$

When $x=0$, all of its terms are positive, each being equal to 1 . Moreover, no two consecutive terms can vanish for the same value of $x$, since by (8) they all would vanish simultaneously. If, then, $x$ traverses the positive or negative half-axis, alterations in the number of changes of sign in (9) can take place only when $x$ passes through a root of $D_{2 n}$. As $x$ approaches $\infty$, the dominant term in $D_{2 n}$ is $b_{2} b_{4} \cdots b_{2 n} x^{n}$. Hence for $x=+\infty$ the number of changes of sign in (9) is equal to the number of negative signs in

$$
b_{2}, b_{4}, \cdots, b_{2 n},
$$

while for $x=-\infty$ it is equal to the number of positive signs. But the number of terms in (10) is exactly equal to the degree of $D_{2 n}$. We have therefore

THEOREM I. - If in the continued fraction (4) $b_{2 n} b_{2 n+1}$ is always positive, the roots of $D_{2 n}$ are real, and the number of positive and of negative roots is equal respectively to the number of negative and of positive terms in (10).

2. The even convergents. - The considerations just adduced show also that the sequence (9) has all the properties of a Sturm series in the positive and negative half axes taken separately. As $x$ passes out from the origin, there is a gain in the number of changes of sign in (9) every time that $x$ passes through a root of $D_{2 n}$. This can only occur if between two consecutive roots of $D_{2 n}$ in each half axis, there is included a root of $D_{2 n-2}$. Hence, since all the roots of $D_{2 n}$ are real, the roots of $D_{2 n-2}$ must be likewise real and alternate with those of $D_{2 n}$ on either side of the origin. It is evident also that $D_{2 n}$ must be the first to vanish after leaving the origin.

The reality and location of the roots of $N_{2 n}$ can next be ascertained. From (8) and the analogous equation for $N_{n}$ we obtain

$$
D_{2 n} N_{2 n-2}-N_{2 n} D_{2 n-2}=b_{2 n-1} b_{2 n-2} x^{2}\left(D_{2 n-2} N_{2 n-4}-N_{2 n-2} D_{2 n-4}\right) ;
$$

whence it follows that

$$
D_{2 n} N_{2 n-2}-N_{2 n} D_{2 n-2}=-b_{2 n-1} b_{2 n-2} \cdots b_{2} x^{2 n-2} .
$$

Let now any two consecutive roots of $D_{2 n}$ which lie on the same side of the origin be substituted in the last equation. Inasmuch as the right hand member is always negative, each root will give to $N_{2 n} D_{2 n-2}$ a positive sign. Since also there 
is included between them one and only one root of $D_{2 n-2}$, it follows that they must enclose one of the $n-1$ roots of $N_{2 n}$. If the roots of $D_{2 n}$ all lie on the same side of the origin, there are $n-1$ such pairs of roots, and the location of the roots of $N_{2 n}$ will therefore have been completely determined. On the other hand, if the roots of $D_{2 n}$ lie on both sides of the origin, the number is $n-2$, and there remains accordingly one root of $N_{2 n}$ whose position is yet to be determined. Now neither of the two intervals between the origin and the adjacent roots of $D_{2 n}$ contains a root of $D_{22_{n-2}}$. A.t the two latter points we know that $N_{2 n} D_{2 n-2}$ is positive, and at the origin it is also positive, being equal to 1 . The remaining root of $N_{2 n}$ therefore cannot be contained in either of these two intervals, and the only place remaining for this root is between one of the two outermost roots of $D_{2 n}$ and the point of infinity.

The results thus obtained can be stated as

TheOREM II.-On each side of the origin the roots of $D_{2 n}$ are separated by the roots of $D_{2 n-2}$ and also by those of $N_{2 n}$, and the root nearest to the origin on either side must be a root of $D_{2 n}$.

3. The odd convergents.-We next consider the odd convergents. Between these and the even convergents there exist the familiar relations:

$$
\begin{aligned}
& D_{2 n} N_{2 n-1}-N_{2 n} D_{2 n-1}=b_{2} b_{3} \cdots b_{2 n} x^{2 n-1}, \\
& D_{2 n+1} N_{2 n}-N_{2 n+1} D_{2 n}=-b_{2} b_{3} \cdots b_{2 n+1} x^{2 n}
\end{aligned}
$$

If in each of these equations two consecutive roots of $D_{2 n}$ are substituted which lie upon the same side of the origin, we conclude, by reasoning in the same manner as in $\S 2$, that they must enclose at least one root of $D_{2 n-1}$ and one of $D_{2 n+1}$ since they enclose a single root of $N_{2 n}$. Now the degrees of $D_{2 n-1}, D_{2 n}$ and $D_{2 n+1}$ are $n-1, n$, and $n$ respectively. Hence the location of all the roots of $D_{2 n-1}$ and $D_{2 n+1}$ will be fixed relatively to those of $D_{2 n}$ with the exception of one root of the former and two of the latter, unless all the roots of $D_{2 n}$ should fall upon the same side of the origin when there would be only a root of $D_{2 n+1}$ to locate.

To fix these roots, consider again the intervals between the origin and the adjacent roots of $D_{2 n}$. The substitution, in turn, of these two roots in (11) gives opposite signs to the right hand member and hence also to $N_{2 n} D_{2 n-1}$. Since neither interval contains a root of $N_{2 n}$, it follows that one of them must contain the remaining root of $D_{2 n-1}$. We conclude accordingly that the roots of $D_{2 n}$ and $D_{2 n-1}$ alternate along the real axis. If the same two roots are sub. stituted in (12), $D_{2 n+1} N_{2 n}$ will have a negative sign in both cases since the right hand member is negative for any real value of $x$. But at the origin $D_{2 n+1} N_{2 n}=1$. Consequently, as neither of the two intervals contains a root of $N_{2 n}$, they must each contain a root of $D_{2 n+1}$.

A similar conclusion holds for 
the single interval terminating in the origin when the roots of $D_{2 n}$ lie on only one side of the origin. This completes the proof of the following result:

THEOREM III.-The roots of $D_{2 n}$ and $D_{2 n-1}$ alternate along the real axis, but the roots of $D_{2 n}$ and $D_{2 n+1}$ alternate only in the positive and negative half axes taken separately. In each half axis there is the same number of roots of $D_{2 n+1}$ as of $D_{2 n}$, and the root adjacent to the origin is always a root of $D_{2 n+1}$.

Having ascertained now the situation of the roots $D_{2 n+1}$, we can next employ (12) for the purpose of finding the location of the roots of $N_{2 n+1}$ with respect to those of $D_{2 n+1}$. On the left hand side of this equation the highest power of $x$ is obtained from the multiplication of the terms of highest degree in $D_{2 n}$ and $N_{2 n+1}$. An inspection of (6) and (7) shows at once that the coefficients of these terms cannot vanish since $b_{n} \neq 0$. Hence the sign of the left hand member of (12) for $x= \pm \infty$ is identical with that of $-N_{2 n+1} D_{2 n}$. But the sign of the right-hand member is always negative, and consequently $N_{2 n+1} D_{2 n}$ is positive both for $x=+\infty$ and for $x=-\infty$. Furthermore, if $x$ in (12) is placed equal to any root of $D_{2 n+1}$, again $N_{2 n+1} D_{2 n}$ will be positive. But we know that between any two consecutive roots of $D_{2 n+1}$ on the same side of the origin and between the outermost root on either side and the point at infinity there lies one and only one root of $D_{2 n}$. It follows that the roots of $N_{2 n+1}$ are situated in the same manner as those of $D_{2 n}$. This result may be recapitulated for convenience of future reference in

Theorem IV. - The roots of $N_{2 n+1}$ and $D_{2 n+1}$ alternate on each side of the origin. On either side there is the same number of roots of one as of the other, and the roots of $D_{2 n+1}$ lie nearer to the origin than the corresponding roots of $N_{2 n+1}$.

To complete the discussion it remains to determine the relative positions of the roots of $N_{n}$ and $N_{n-1}$. This also can be done with the aid of (11) and (12). It will be found that the roots of $N_{2 n}$ and $N_{2 n-1}$ alternate along the axis while the roots of $N_{2 n}$ and $N_{2 n+1}$ alternate only on the two sides of the origin considered separately, the roots adjacent to the origin being thoso of $N_{2 n+1}$.

As it will be necessary subsequently to treat the odd convergents apart from the even, it is desirable to know also the relative positions of the roots of the denominators of two consecutive odd convergents. This can be inferred with the aid of theorem III from the equation

$$
D_{2 n+1}=D_{2 n}+b_{2 n+1} x D_{2 n-1} \text {. }
$$

By substituting pairs of consecutive roots of $D_{2 n+1}$ we obtain

Theorem V.-The roots of $D_{2 n+1}$ and $D_{2 n-1}$ alternate with each other along the axis of $x$. 
4. On the relative situation of the roots of $D_{n}$ and $D_{n+m}$ - - We proceed next to compare $D_{n}$ and $D_{n+m}$. Let $b_{n+1} x N_{m}^{(n)} / D_{m}^{(n)}$ denote the $m$ th convergent of the continued fraction obtained by omitting the first $n$ partial fractions in (4). Then obviously

$$
D_{n+m}=D_{m}^{(n)} D_{n}+b_{n+1} x N_{m}^{(n)} D_{n-1},
$$

which may be written in the form

$$
\frac{D_{n+m}}{b_{n+1} x D_{n} N_{m}^{(n)}}=\frac{D_{m}^{(n)}}{b_{n+1} x N_{m}^{(n)}}+\frac{D_{n-1}}{D_{n}} .
$$

Suppose now that $n$ and $m$ are even integers. By the preceding theorems the zeros and infinities of each fraction in the right hand member of (14) are real and separate each other. But when this is the case, it follows from a well known theorem that the fraction either always increases or always decreases when $x$ describes the real axis in a positive direction.

To ascertain whether there is an increase or a decrease of our two fractions, let $m$ and $n$ be replaced by $2 n$ and $2 m$ and consider then the signs of the derivatives of the fractions:

$\frac{D_{2 n} D_{2 n-1}^{\prime}-D_{2 n}^{\prime} D_{2 n-1}}{\left(D_{2 n}\right)^{2}}, \frac{b_{2 n+1} x N_{2 m}^{(2 n)} \frac{d D_{2 m}^{(2 n)}}{d x}-b_{2 n+1} N_{2 m}^{(2 n)} D_{2 m}^{(2 n)}-b_{2 n+1} x D_{2 m}^{(2 n)} \frac{d N_{2 m}^{(2 n)}}{d x}}{\left(b_{2 n+1} x N_{2 n}^{(2 n)}\right)^{2}}$

For $x=0$ the numerator of the first fraction reduces to $-b_{2 n}$ (see equations $(6)$ and $(7))$ and that of the second fraction to $-b_{2 n+1}$. Since by hypothesis $b_{2 n}$ and $b_{2 n+1}$ have like signs, the two fractions will increase with increasing $x$ if $b_{2 n}$ is negative or decrease if $b_{2 n}$ is positive. A fortiori the left hand member of (14) will be an increasing or a decreasing function respectively for the entire length of the real axis. Now we know that the zeros and infinities of the left hand member are real. It follows that the zeros and infinities must alternate with one another, or, in other words, that the roots of $D_{2 n+2 m}$ and $x D_{2 n} N_{2 m}^{(2 n)}$ separate each other.

A parallel course of reasoning applies to prove the remaining conclusions stated in the following theorem:

TheOREM VI.- The roots of $D_{2 n+2 m}$ are separated by those of $x D_{2 n} N_{2 n}^{(2 n)}$ and by those of $D_{2 n-1} D_{2 m}^{(2 n)}$. So also the roots of $D_{2 n+2 n+1}$ alternate with those of $x D_{2 n} N_{2 m+1}^{(2 n)}$ and with those of $D_{2 n-1} D_{2 m+1}^{(2 n)}{ }^{*}$

It should be pointed out that this theorem is not to be so interpreted as to preclude the possibility of the coincidence of two adjacent roots of the pair of functions compared. As the $b_{i}$ vary in value, it may happen that two such

\footnotetext{
* StreltJes $(\$ 5)$ demonstrates this theorem for his continued fraction in another manner, but the proof just given is simpler.
} 
roots momentarily unite. Suppose, for example, a root of $N_{2 m}^{(2 n)}$ to coincide with one of $D_{2 n}$. Then it must also be a root of $D_{2 n+2 m}$, inasmuch as

$$
D_{2 n+2 m}=D_{2 m}^{(2 n)} D_{2 n}+b_{2 n+1} x N_{2 m}^{(2 n)} D_{2 n-1} \text {. }
$$

Since, moreover, it is impossible for a root of $D_{2 n}$ to coincide with one of $D_{2 n-1}$ or a root of $D_{2 m}^{(2 n)}$ with one of $N_{2 m}^{(2 n)}$, this is the only case in which a root of $D_{2 n+2 m}$ unites with a root of $x D_{2 n} N_{2 m}^{(2 n)}$.

Equation (15) shows that when this coincidence takes place, the root of $D_{2 n+2 m}$ is entirely independent of the value of $b_{2 n+1}$. A like independence exists when a root coincides with a root of $D_{2 m}^{(2 n)}$ and of $D_{2 n-1}$, and in no other case.

5. On the condensation of the roots.-We proceed next to consider the possibility of the accumulation of the roots of $D_{n}$ in the vicinity of a point of the real axis when $n$ increases indefinitely. Let $P$ be any given point except the origin, and suppose first that the number of roots of $D_{n}$ in the vicinity of $P$ has no upper limit. Theorem VI shows that between any two consecutive roots of $D_{n}$ on the same side of the origin there must always lie at least one root of $D_{n+m}$. Our hypothesis therefore leads at once to the conclusion that the number of roots of $D_{n}$ within a prescribed distance of $P$ will become and remain greater than any prescribed integer, if $n$ be sufficiently increased.

Suppose, on the other hand, that the number of roots of $D_{n}$ in the vicinity of $P$ has an upper limit $l$. Then within any sufficiently small interval which contains $P$ as an interior point, there will be exactly $l$ roots of some $D_{n}$, while each succeeding $D_{n+m}$ will have either $l$ or $l-1$ roots in the interval. Choose now for consideration such a group of $l$ roots. If they lie on both sides of $P$, let $\gamma^{\prime}, \gamma^{\prime \prime}$ denote the two roots adjacent to it. By increasing $n$ another group of $l$ roots of some succeeding $D_{n+m}$ will be obtained which lie within $\left(\gamma^{\prime}, \gamma^{\prime \prime}\right)$. But if $l>2$, this will leave two roots of the former group which include between them no root of the latter, which is impossible. On the other hand, if all the roots of the first group lie on the same side of $P$, a second group of $l$ roots will ultimately be included between $P$ and the nearest root of the first group. This will be impossible unless $l=1$. We conclude therefore that when $n$ increases indefinitely, either an unlimited number of roots of $D_{n}$ will accumulate in the vicinity of $P$ or the number will be equal at most to 2 ; and if it is equal to 2 , the two roots which approach $P$ must lie on opposite sides of this point.

The same reasoning is equally applicable to the accumulation of positive (negative) roots of $D_{n}$ in the vicinity of the origin. Either the number of roots thus accumulating will be infinite or it will not exceed 1 . We shall supplement this result presently by showing that if $b_{2 n-1}+b_{2 n}$ has no lower limit, an infinite number of positive roots will condense in the vicinity of the origin. 
6. On the limiting positions of the roots.-Let the roots of $D_{2 n}$ now be numbered on each side of the origin in the order in which they are met in proceeding out from this' point, those on the positive side being denoted by $x_{1}^{\prime}, x_{2}^{\prime}, \ldots$ and those on the opposite side by $x_{1}^{\prime \prime}, x_{2}^{\prime \prime}, \ldots$. From theorem II it is clear that as $n$ increases, $x_{i}^{\prime}$ and $x_{i}^{\prime \prime}$ move always toward the origin. They have, then, definite limiting positions, but the limiting positions of $x_{i}^{\prime}\left(\right.$ or $\left.x_{i}^{\prime \prime}\right)$ for different values of $i$ are not necessarily distinct. It follows, however, from $\$ 5$ that if two roots, $x_{i}^{\prime}$ and $x_{i+1}^{\prime}$, have a common limit, this must also be the limit of all the succeeding roots.

When there is an upper limit to the number of roots of $D_{n}$ on either side of the origin, as in the case considered by STIELTJES, analogous conclusions can be drawn for the roots of $D_{2 n+1}$. Suppose, for example, that the number of roots on the negative side is limited. When once the number of negative roots of $D_{2 n+1}$ reaches its maximum, it must remain equal to this maximum for all succeeding values of $n$ because the number of negative roots of $D_{2 n+1}$, as of $D_{2 n}$, is equal to the number of positive signs in the sequence (10). Now the roots of $D_{2 n+1}$ are not only separated by those of $D_{2 n+3}$ but are included between the extreme roots of the latter. As soon, therefore, as the number of negative roots has attained its maximum, each negative root $\bar{x}_{i}^{\prime \prime}$ with a further increase of $n$ must recede from the origin while the $i$ th positive root $\bar{x}_{i}^{\prime}$ will approach it. Hence there is a limiting position for the $i$ th root on either side of the origin. If the limits of two consecutive positive roots coincide, all the succeeding positive roots have the same limit, but the negative roots, being restricted in number, must have distinct limits, since two consecutive roots of $D_{n}$ on the same side of the origin will include between them at least one root of each $D_{n+m}$.

When the roots of $D_{2 n}$ and $D_{2 n+1}$ are enumerated in the opposite order-that is, in the order in which they occur in going from the point at infinity toward the origin - we shall denote those on the positive side by $y_{i}^{\prime}$ and $\bar{y}_{i}^{\prime}$ respectively and those on the opposite side by $y_{i}^{\prime \prime}$ and $\bar{y}_{i}^{\prime \prime}$. Since the roots of $D_{2 n+3}$ separate and enclose those of $D_{2 n+1}$, the $i$ th roots $\bar{y}_{i}^{\prime}, \bar{y}_{i}^{\prime \prime}$ move with increasing $n$ toward the point at infinity, and each has a limiting position. Corresponding conclusions can be made for the roots of $D_{2 n}$ if its positive or negative roots are restricted in number.

7. Conditions for the indefinite approach of roots to the origin. The importait question now naturally arises : what is the necessary and sufficient condition that the roots of $D_{n}$ with increasing $n$ shall approach infinitesimally near to the origin? This question was examined by StiEltJes* for continued fractions with positive coefficients, and he found the condition to be that $b_{n}$ should have no upper limit. Further, I have shown $\dagger$ that if $\left|b_{n}\right|$ has an upper limit $L$ in

* Loc. cit., 8 8 9, 10.

†Transactions of the American II athematical Society, vol. $2(: 901)$, p. $477, \& 2$. 
any continued fraction whatsoever of the form (4), whether with real or complex coefficients, the numerators and denominators of the convergents can not vanish in the interior of a circle having its center in the origin and a radius equal to $1 / 4 L$. Therefore a necessary condition for the approach of roots to within less than any assignable distance of the origin is that $\left|b_{n}\right|$ shall have no upper limit. We shall consider now the question of the sufficiency of this condition for the class of continued fractions treated in this paper, adhering here as far as possible to the methods of Stieltues.

Let us take first the hypothesis that $\left|b_{2 n+1}\right|$ has no upper limit. By a previous theorem $D_{2 n}$ and $D_{2 n+1}$ have on either side of the origin the same number of roots. Also from (6) and (7)

$$
\begin{aligned}
& \sum\left(\frac{1}{{\overline{x_{i}^{\prime}}}_{i}^{\prime}}\right)+\sum\left(\frac{1}{\overline{\bar{x}_{i}^{\prime \prime}}}\right)=-\left(b_{1}+b_{2}+\cdots+b_{2 n+1}\right), \\
& \sum\left(\frac{1}{x_{i}^{\prime}}\right)+\sum\left(\frac{1}{{\overline{x_{i}^{\prime \prime}}}^{\prime \prime}}\right)=-\left(b_{1}+b_{2}+\cdots+b_{2 n}\right) ;
\end{aligned}
$$

whence it follows that

$$
\sum\left(\frac{1}{\overline{\bar{x}}_{i}^{\prime}}-\frac{1}{x_{i}^{\prime}}\right)+\sum\left(\frac{1}{\bar{x}_{i}^{\prime \prime}}-\frac{1}{x_{i}^{\prime \prime}}\right)=-b_{2 n+1} .
$$

If $b_{2 n+1}$ is negative, let the last equation be written in the form

$$
\frac{1}{\overline{x_{1}^{\prime}}}=-b_{2 n+1}+\sum\left(\frac{1}{x_{i}^{\prime \prime}}-\frac{1}{\bar{x}_{i}^{\prime \prime}}\right)+\sum_{i=1}^{k-1}\left(\frac{1}{\bar{x}_{i}^{\prime}}-\frac{1}{\bar{x}_{i+1}^{\prime}}\right)+\frac{1}{x_{k}^{\prime}}
$$

in which $x_{k}^{\prime}$ denotes the largest positive root of $D_{2 n}$. Each of the binomial terms on the right hand side is positive because of the mode of alternation of the roots of $D_{2 n}$ and $D_{2 n+1}$ (theorem III). It follows that $1 / \bar{x}_{1}^{\prime}>-b_{2 n+1}$. Consequently, if there is a set of negative coefficients $b_{2 n+1}$ whose absolute values have no upper limits, the smallest positive root of $D_{2 n+1}$ will approach within less than any assigned distance of the origin. In exactly the same manner it can be shown that the first negative root of $D_{2 n+1}$ will come indefinitely near to the origin if the positive coefficients $b_{2 n+1}$ have no upper limit.

Suppose next that $\left|b_{2 n+2}\right|$ has no upper limit while $\left|b_{2 n+1}\right|$ has. If the roots of $D_{2 n+2}$ on the two sides of the origin are denoted in order by $v_{i}^{\prime}, v_{i}^{\prime \prime}$, one and only one of these roots - call it $v_{k}$ - lies further away from the origin than any root of $D_{2 n}$ on the same side. To every other root $v$ there corresponds one of $D_{2 n}$, and the equation analogous to $(16)$ which connects the two systems of roots is

$$
\sum\left(\frac{1}{v_{i}^{\prime}}-\frac{1}{x_{i}^{\prime}}\right)+\sum\left(\frac{1}{v_{i}^{\prime \prime}}-\frac{1}{x_{i}^{\prime \prime}}\right)+\frac{1}{v_{k}}=-\left(b_{2 n+1}+b_{2 n+2}\right) \text {. }
$$


By theorem I the sign of $v_{k}$ will be opposite to that of $b_{2 n+2}$. If, then, there is any set of negative coefficients $b_{2 n+2}$ whose absolute values have no upper limit, we shall rearrange the terms of the last equation as follows :

$$
\frac{1}{v_{1}^{\prime}}=-\left(b_{2 n+1}+b_{2 n+2}\right)+\sum_{i=1}^{k-2}\left(\frac{1}{x_{i}^{\prime}}-\frac{1}{v_{i+1}^{\prime}}\right)+\sum\left(\frac{1}{x_{i}^{\prime \prime}}-\frac{1}{v_{i}^{\prime \prime}}\right)+\left(\frac{1}{x_{k-1}^{\prime}}-\frac{1}{v_{k}}\right) .
$$

By theorem II all the binomial terms after the first will be positive; hence

$$
\frac{1}{v_{1}^{\prime}}>-\left(b_{2 n+1}+b_{2 n+2}\right) \text {. }
$$

But by hypothesis there is some set of values of $n$ for which the right hand member of this inequality is positive and has no upper limit. It follows that $v_{1}^{\prime}$ must come within any assigned distance of the origin, and as it diminishes with $n$, this point must be its limit. The same conclusion will hold also for the first positive root of $D_{2 n+3}$ since the root lies between the origin and $v_{1}^{\prime}$. Parallel reasoning shows that the first negative root of $D_{2 n}$ and of $D_{2 n+1}$ has the origin as a limit whenever there is any set of values for which $b_{2 n+1}+b_{2 n+2}$ is positive and has no upper limit.

It has thus been established that if $\left|b_{n}\right|$ has no upper limit, either the first positive or first negative root of $D_{2 n+1}$, or both, will come indefinitely near to the origin, though this point need not always be a limit. The suppositions made in the two preceding paragraphs concerning the even and odd elements $b_{2 n}$, $b_{2 n+1}$ are obviously not affected by the omission of an even number of partial fractions at the outset of (4). The same conclusions therefore are valid for $D_{2 m+1}^{(2 n)}$ and $D_{2 m}^{(2 n)}$ as for $D_{2 n+1}$ and $D_{2 n+2}$. Accordingly, if there is any set of values of $n$ for which $b_{2 n-1}+b_{2 n}$ is negative (positive) and has no upper limit, the first positive (negative) roots of $D_{2 n-1}$ and $D_{2 m+1}^{(2 n)}$ will not only come within any assigned distance of the origin but will remain there if $m$ and $n$ are sufficiently great.

Suppose then that there is such a set of values for which $b_{2 n-1}+b_{2 n}$ is negative. The same then is true either of $b_{2 n}$ or of $b_{2 n-1}$; and if true of the latter, it is true also of the former, since $b_{2 n-1}$ and $b_{2 n-2}$ have like signs. We may therefore assume that there is an infinite sub-set of values for which $b_{2 n}$ also is negative.

By theorem VI the $n+m$ roots of $D_{2 n+2 m+1}$ alternate with $n+m-1$ roots of $D_{2 n-1} D_{2 m+1}^{(2 n)}$. Hence on one side of the origin or the other the nearest root will belong to $D_{2 n+2 m+1}$, and this side will be the one on which there lie more roots of this polynomial than of $D_{2 n-1} D_{2 m+1}^{(2 n)}$. Now the number of positive roots of $D_{2 n-1}$ and of $D_{2 n+2 m+1}$ is equal to the number of negative signs occurring in the first $n-1$ and $n+m$ terms of the sequence

$$
b_{2}, b_{4}, b_{6}, \cdots,
$$


while the number of positive roots of $D_{2 m+1}^{2 n)}$ is equal to the number of negative signs in the first $n$ terms of the sequence subsequent to $b_{2 n}$. Consequently, when $b_{2 n}$ is negative, the first positive root of $D_{2 n+2 n+1}$ lies nearer to the origin than the first positive root of $D_{2 n-1} D_{2 m+1}^{(2 n)}$. Since also the two systems of roots alternate, we conclude that two positive roots of $D_{2 n+2 m+1}$ must come and remain within any assigned distance of the origin. This conclusion can now be transferred to $D_{2 n-1}$ and $D_{2 m+1}^{2 n n}$ if $n$ and $m$ are sufficiently great. By repeating then the reasoning we next obtain three positive roots of $D_{2 n+2 m+1}$ within the assigned distance of the origin, and so on. As also its roots are separated by those of $D_{2 n+2 n}$, we see finally that the origin must be a point of condensation * of an infinite number of positive roots of $D_{n}$, if there is any set of values for which $b_{2 n-1}+b_{2 n}$ is negative and has numerically no upper limit.

The result thus reached may be recapitulated as follows:

TheOREM VII.-The necessary and sufficient condition that the distance between the origin and the nearest root of $D_{n}$ shall have no lower limit is that $\left|b_{n}\right|$ shall have no upper limit. If there is any set of values of $n$ for which $b_{2 n-1}+b_{2 n}$ has numerically no upper limit and is negative (positive), an infinitely increasing number of positive (negative) roots of $D_{n}$ will accumulate with increasing $n$ in the vicinity of the origin.

8. The special case in which $\lim b_{n}=0$. - There seems to be no simple test for the indefinite increase of the outermost root or roots of $D_{n}$. A sufficient condition, however, is that $\left|b_{2 n}\right|$ shall have no lower limit. 'This can be inferred from the fact that $-b_{2 n}$ is the quotient of the product of the roots of $D_{2 n-2}$ by those of $D_{2 n}$. For since the roots $x_{i}^{\prime}, x_{i}^{\prime \prime}$ of $D_{2 n}$ lie nearer to the origin than the corresponding roots of $D_{2 n-2}$ and since the former polynomial has one more root than the latter, the distance of this extra root from the origin must for some value of $n$ exceed any assigned quantity if $\left|b_{2 n}\right|$ has no lower limit.

A special and important case which I shall examine is that in which $b_{n}$ has the limit 0 . In a preceding paper $\dagger I$ have given a very simple proof that when $\lim b_{n}=0$ in any continued fraction of the form (4), the continued fraction represents a meromorphic function and converges in all the points of the $x$-plane with the exception of the poles of this function. As $n$ increases, the roots of $D_{n}$ which lie within any given finite distance of the origin approach limiting positions, and these positions are the poles of the function.

I shall supplement this result for the continued fractions of the present paper by showing, conversely, when it represents a meromorphic function, $\lim b_{n}=0$. In proving this we may start with the hypothesis, apparently less restrictive, that the roots $\bar{x}_{i}^{\prime}, \bar{x}_{i}^{\prime \prime}$ of $D_{2 n+1}$ with increasing $n$ approach limits and that these

* The reasoning by which the existence of the point of condensation is established could be applied to the continued fraction of Srielt.Jes but is not used hy him.

†Transactions of the American Mathematical Society, vol. 2 (1901), p. 476.

Trans. Am. Math. Soc. 21 
limits are distinct from one another and from 0 . Let the roots of $D_{2 n-1}$ be denoted by $\bar{v}_{i}^{\prime}, \bar{v}_{i}^{\prime \prime}$. These roots alternate with those of $D_{2 n+1}$, and on one side of the origin there is one more root of the latter polynomial than of the former. Let $\bar{x}_{k}$ be the outermost root on this side. Then we have

$$
\sum\left(\frac{1}{{\overline{x_{i}^{\prime}}}_{i}^{\prime}}-\frac{1}{{\overline{v_{i}^{\prime}}}_{i}^{\prime}}\right)+\sum\left(\frac{1}{{\overline{x_{i}^{\prime \prime}}}_{i}^{\prime}}-\frac{1}{\overline{\bar{v}}_{i}^{\prime \prime}}\right)+\frac{1}{\bar{x}_{k}}=-\left(b_{2 n}+b_{2 n+1}\right) .
$$

Choose now any interval $(s,-s)$ of the axis of $x$ so large that $1 / s$ shall be less than any arbitrarily prescribed quantity $\epsilon / 4$. In consequence of the assumption of limiting positions for the roots the differences $\left|1 / \bar{x}_{i}^{\prime}-1 / \bar{v}_{i}^{\prime}\right|$ and $\left|1 / \bar{x}_{i}-1 / \bar{v}_{i}^{\prime \prime}\right|$ can be made as small as we wish for all pairs of roots which lie within the segment, and as the number of such pairs is finite, the sum of these differences can be made less then $\epsilon / 4$. Consider next the positive roots outside of the interval. We have

$$
\left|\frac{1}{\overline{\bar{x}}_{j}^{\prime}}-\frac{1}{\overline{\bar{v}}_{j}^{\prime}}+\frac{1}{{\overline{x_{j+1}^{\prime}}}_{j+1}^{\prime}}-\frac{1}{\overline{\bar{v}}_{j+1}^{\prime}}+\cdots-\frac{1}{\overline{\bar{v}}_{j+*}^{\prime}}\right|<\frac{1}{\left|\overline{\bar{x}_{j}^{\prime}}\right|}<\epsilon / 4
$$

since the terms are alternately positive and negative and each less than the preceding owing to the alternation of the roots. A similar inequality holds for the negative roots exterior to the interval. Lastly, if $n$ is sufficiently large, $1 /\left|x_{k}\right|<\epsilon / 4$. It follows therefore from (17) that we may make

$$
\left|b_{2 n}+b_{2 n+1}\right|<\epsilon .
$$

Hence, as $b_{2 n}$ and $b_{2 n+1}$ have like signs, $\lim b_{n}=0$. But this is what we wished to prove.

A comparison between the roots of $D_{2 n-1}$ and $D_{2 n}$ next gives

$$
\left|\sum\left(\frac{1}{x_{i}^{\prime}}-\frac{1}{\bar{v}_{i}^{\prime}}\right)+\sum\left(\frac{1}{x_{i}^{\prime \prime}}-\frac{1}{\bar{v}_{i}^{\prime \prime}}\right)+\frac{1}{x_{k}}\right|=\left|-b_{2 n}\right|<\epsilon .
$$

The binomial terms here have all the same sign as $1 / x_{k}$ because of the alternation of the roots of the two polynomials. Consequently we have

$$
\left|\frac{1}{x_{i}^{\prime}}-\frac{1}{\bar{v}_{i}^{\prime}}\right|<\epsilon, \quad\left|\frac{1}{x_{i}^{\prime \prime}}-\frac{1}{\overline{\bar{v}}_{i}^{\prime \prime}}\right|<\epsilon,
$$

and the limiting positions of the roots of $D_{2 n}$ therefore are identical with those of $D_{2 n-1}$.

From the identity of these positions the convergence of the continued fraction can be argued. The manner in which this is done is indicated in $\S 14$. 
II. Derivation of the integral $\int_{a}^{b} \frac{d \Phi(u)}{z+u}$.

9. Change of variable. - It will be convenient now to make the substitution $x=1 / z$. Our continued fraction (4), after the removal of a factor $z$, can then be thrown into the form

$$
\frac{1}{z}+\frac{b_{1}}{1}+\frac{b_{2}}{z}+\frac{b_{3}}{1}+\frac{b_{4}}{z}+\cdots
$$

If its convergents are denoted by $N_{2 n}(z) / D_{2 n}(z)$ and $N_{2 n+1}(z) / z D_{2 n+1}(z)$, we have

$$
D_{n}(z)=z^{n_{1}} D_{n}(x), \quad N_{n}=z^{n_{2}} N_{n}(x),
$$

in which $n_{1}$ and $n_{2}$ denote the degrees of $D_{n}(x)$ and $N_{n}(x)$. It should be noticed that the coefficients of the highest powers of $z$ in $D_{n}(z)$ and $N_{n}(z)$ are equal to 1 . No confusion will result if hereafter we abbreviate these symbols by $D_{n}$ and $N_{n}$, as the context will show whether the argument $z$ or $x$ is intended.

The modifications to be made in the theorems of Section I owing to the exchange of the origin and point at infinity in the transformation $z=1 / x$ are too simple to require statement. We-merely call attention to the fact that the roots of $D_{2 n}$ and $N_{2 n}$, also of $z D_{2 n+1}$ and $N_{2 n+1}$ will alternate along the entire axis of z. Decomposition into partial fractions therefore gives

$$
\begin{array}{r}
\frac{N_{2 n}}{D_{2 n}}=\frac{M_{1}}{z+z_{1}}+\frac{M_{2}}{z+z_{2}}+\cdots+\frac{M_{n}}{z+z_{n}} \quad\left(z_{1}<z_{2}<\cdots<z_{n}\right), \\
\frac{N_{2 n+1}}{z D_{2 n+1}}=\frac{N_{1}}{z+z_{1}^{\prime}}+\frac{N_{2}}{z+z_{2}^{\prime}}+\cdots+\frac{N_{0}}{z}+\cdots+\frac{N_{n}}{z+z_{n}^{\prime}}
\end{array}
$$

in which $M_{i}$ and $N_{i}$ are positive and

$$
\left(z_{1}^{\prime}<z_{2}^{\prime}<\cdots<0<\cdots<z_{n}^{\prime}\right),
$$

$$
1=M_{1}+M_{2}+\cdots+M_{n}=N_{1}+N_{2}+\cdots+N_{0}+\cdots+N_{n} .
$$

10. The function $\phi_{n}(u)$.-We now shall consider the even and the odd convergents separately and, after the precedent of STIELTJEs, construct for every even convergent a monotone function $\phi_{n}$ of a real variable $u$ in the following manner :

$$
\begin{aligned}
& \phi_{n}(u)=0, \\
& -\infty<u<z_{1} \text {; } \\
& \phi_{n}(u)=M_{1} \text {, } \\
& z_{1} \leqq u<z_{2} \text {; } \\
& \phi_{n}(u)=M_{1}+M_{2} \text {, } \\
& z_{2} \leqq u<z_{3} \text {; } \\
& \phi_{n}(u)=M_{1}+M_{2}+\cdots+M_{n-1}, \\
& z_{n-1} \leqq u<z_{n} ; \\
& \phi_{n}(u)=M_{1}+M_{2}+\cdots+M_{n} \text {, } \\
& z_{n} \leqq u<+\infty \text {. }
\end{aligned}
$$


The relation which subsists between any two functions thus constructed is fundamental for the formation of an integral which shall represent the limit of the continued fraction, when convergent. Since $\phi_{n+m}(u)$ and $\phi_{n}(u)$ are both increasing functions, their difference can change sign but once between two consecutive roots of $D_{n}(-z)$. After the last root $z_{n}$ such a change is impossible because $\phi_{n+m}(u) \leqq \sum M_{i}=\phi_{n}\left(z_{n}\right)$. Hence the maximum number of changes of sign is $2 n-1$. This number will be reached if, and only if, $\phi_{n+m}(z)-\phi_{n}(z)$ is negative for each of the $n$ roots $z_{i}$ of $D_{n}(-z)$ and is positive for a (smaller) value of $z$ just short of $z_{i}$. We will show this to be the case.*

From (14) and the analogous equation connecting $N_{n+m}(x)$ and $N_{n}(x)$ there results the equation

or

$$
\frac{1}{b_{2 n+1} x}\left(\frac{N_{2 n+2 n}}{N_{2 n} N_{2 m}^{(2 n)}}-\frac{D_{2 n+2 m}}{D_{2 n} N_{2 m}^{(2-\bar{n})}}\right)=\frac{N_{2 n-1}}{N_{2 n}}-\frac{D_{2 n-1}}{D_{2 n}}
$$

$$
\frac{N_{2 n+2 m}}{\bar{D}_{2 n+2 m}}-\frac{N_{2 n}}{D_{2 n}^{n}}=\frac{b_{2 n+1} x N_{2 m}^{(2 n)}\left(D_{2 n} N_{2 n-1}-N_{2 n} D_{2 n}\right)}{D_{2 n} D_{2 n+2 m}}=\frac{b_{2} b_{3} \cdots b_{2 n+1} x^{2 n} N_{2 m}^{(2 n)}}{D_{2 n} D_{2 n+2 m}}
$$

Hence, changing to the variable $z$, we obtain

$$
\begin{aligned}
\left(\frac{M_{1}^{(n+m)}}{z+z_{1}^{(n+m)}}+\cdots+\frac{M_{n+m}^{(n+m)}}{z+z_{n+m}^{(n+m)}}\right)-\left(\frac{M_{1}}{z+z_{1}}\right. & \left.+\cdots+\frac{M_{n}}{z+z_{n}}\right) \\
& =\frac{b_{2} b_{3} \cdots b_{2 n+1} \Pi\left(z+w_{i}\right)}{\Pi\left(z+z_{i}^{(n+m)}\right) \Pi\left(z+z_{i}\right)},
\end{aligned}
$$

in which $z_{i}^{(n+m)}$ and $w_{i}$ denote in increasing order of magnitude the roots of $D_{2 n+2 m}(-z)$ and $N_{2 m}^{(2 n)}(-\dot{z})$ respectively.

Without altering in any manner the form of $(22)$ the $\operatorname{roots} z_{i}, z_{i}^{(n+m)}$, and $w_{i}$ can be brought by a change of origin into the positive half of the axis of $z$. We will suppose for the moment that this has been done.

By theorem VI the $n+m-1$ roots $z_{i}, . w_{i}$ alternate with the $n+m$ roots of $D_{2 n+2 m}(-z)$, and any particular root $z_{j}$ of $D_{2 n}(-z)$ must be followed and preceded immediately by a root of $D_{2 n+2 m}(-z)$. Let $z_{k}^{(n+m)}$ denote the succeeding root. Then within a circular ring having the origin as a center and the inner and outer radii

$$
R=z_{j}, \quad R^{\prime}=z_{i}^{(n+m)},
$$

the two members of $(22)$ can be expanded into a LaUnent's power series $P_{1}(z)+P_{2}(1 / z)$. The coefficient of $1 / z$ on the left hand side of the equation is obviously

* STIELTJES establishes the fact with the aid of certain integrals of a simple nature (loc. cit., 8 42). The proof with slight modifications is also applicable here, but I have preferred to give a new proof based directly upon the relation between the convergents. 


$$
\sum_{i=1}^{k-1} M_{i}^{(n+m)}-\sum_{i=1}^{j} M_{i}=\phi_{n+m}\left(z_{j}\right)-\phi_{n}\left(z_{j}\right),
$$

which is exactly the expression whose sign is desired. We shall determine its sign by seeking the coefficient of $1 / z$ on the right hand side. There the positive product $b_{2} b_{3} \cdots b_{2 n+1}$ can be neglected. Replace momentarily $z$ by $-z$. If any factor of the denominator is denoted by $z-z^{\prime}$, its expansion into a series gives

$$
\frac{1}{z-z^{\prime}}=-\frac{1}{z^{\prime}}\left(1+\frac{z}{z^{\prime}}+\frac{z^{2}}{\left(z^{\prime}\right)^{2}}+\cdots\right)
$$

or

$$
\frac{1}{z-z^{\prime}}=\frac{1}{z}\left(1+\frac{z^{\prime}}{z}+\frac{\left(z^{\prime}\right)^{2}}{z^{2}}+\cdots\right)
$$

according as $z^{\prime} \geqq R^{\prime}$ or $z^{\prime} \leqq R$. All the terms within the parentheses have positive coefficients since $z^{\prime}$ has been brought to the positive side of the origin. The corresponding expansions for the factors of the numerator on the right hand side of $(22)$ are

$$
\begin{array}{rr}
-w^{\prime}\left(1-\frac{z}{w^{\prime}}\right) & \left(w^{\prime}>R^{\prime}\right), \\
z\left(1-\frac{w^{\prime}}{z}\right) & \left(w^{\prime}<R\right) .
\end{array}
$$

Let $z^{\prime \prime}$ be the root of $D_{2 n+2 m}(-z)$ which immediately precedes any assigned root $w^{\prime}$ of $N_{2 m}^{(2 n)}(-z)$ and $z^{\prime \prime \prime}$ the root which immediately follows it. If $w^{\prime}>R^{\prime}$, multiply (25) into the expansion (23) for which $z^{\prime}=z^{\prime \prime}$, and if $w^{\prime}<R$, multiply (26) into the expansion (24) for which $z^{\prime}=z^{\prime \prime \prime}$. Since $z^{\prime \prime}<w^{\prime}<z^{\prime \prime \prime}$, the product of the two parentheses in either case will be a series with positive coefficients, and the continued multiplication of all such products gives a Laurent's power series, the coefficients of which are positive. Consequently we need to attend only to the product of the constant terms without the parentheses. Now these terms, $-1 / z^{\prime}$ and $-w^{\prime}$, are negative and correspond to roots of $N_{2 m}^{(2 n)}(-z), D_{2 n}(-z)$, and $D_{2 n+2 m}(-z)$ which are equal to or greater than $R^{\prime}$. As the total number of such roots is odd in consequence of the alternation of the roots of $D_{2 n+2 m}$ and $D_{2 n} N_{2 m}^{(2 n)}$, we come finally to the conclusion that the coefficient of $1 / z$ in the Laurentian expansion of the right hand member of (22) must be negative. In other words $\phi_{n+m}\left(z_{i}\right)-\phi_{n}\left(z_{i}\right)$ is negative, as was to be proved.

If a LaURent's power series for (22) is formed for values of $z$ slightly less than $z_{j}$, it will be valid for a certain circular ring whose outer radius is $R=\left|z_{j}\right|$, and the number of expansions of the form (23) and (25) will be less by 1 than before. Hence $\phi_{n+m}(z)-\phi_{n}(z)$ will positive. This completes the 
proof that $\phi_{n+m}(u)-\phi_{n}(u)$ changes sign the maximum number of times, once at each root of $D_{2 n}$ (except the last) and once between any two of its roots which are consecutive.

11. The function $\psi_{n}(u)$. - For each odd convergent $N_{2 n+1} / z D_{2 n+1}$ a monotone function $\psi_{n}(u)$ can be similarly constructed having its discontinuities in the roots $z_{i}^{\prime}$ of $D_{2 n+1}(z)$. Thus

$$
\begin{array}{lr}
\psi_{n}(u)=0, & -\infty<u<z_{1}^{\prime} ; \\
\psi_{n}(u)=N_{1}, & z_{1}^{\prime} \leqq u<z_{2}^{\prime} ; \\
\psi_{n}(u)=N_{1}+N_{2}, & z_{2}^{\prime} \leqq u<z_{3}^{\prime} ;
\end{array}
$$

To compare $\psi_{n+m}(u)$ and $\psi_{n}(u)$ use may be made of the relation

$$
\frac{N_{2 n+2 m+1}}{z D_{2 n+2 m+1}}-\frac{N_{2 n+1}}{z D_{2 n+1}}=-\frac{b_{2} b_{3} \cdots b_{2 n+2} N_{2 m}^{(2 n+1)}}{z D_{2 n+1} D_{2 n+2 m+1}}
$$

Since by theorem VI the roots of $D_{2 n+2 m+1}(z)$ alternate with those of

$$
z D_{2 n+1}(z) D_{2 m-1}^{(2 n+2)}(z) \equiv z D_{2 n+1}(z) N_{2 m}^{(2 n+1)}(z),
$$

it follows by reasoning like that of $\S 10$ that $\psi_{n+m}\left(z_{i}^{\prime}\right)-\psi_{n}\left(z_{i}^{\prime}\right)$ has a constant sign for all roots of $D_{2 n+1}$ and changes sign when $u$ passes through each root excepting either the first or the last. Since also $\psi_{n+m}(u)$ and $\psi_{n}(u)$ are increasing functions, $\psi_{n+m}\left(z_{i}^{\prime}\right)-\psi_{n}\left(z_{i}^{\prime}\right)$ must be negative.

We may add that $\psi_{n+m}\left(z_{i}\right)-\phi_{n}\left(z_{i}\right)$ and $\phi_{n+m}\left(z_{i}^{\prime}\right)-\psi_{n}\left(z_{i}^{\prime}\right)$ are both negative.

12. First special case.-The relations established in $\S \S 10-11$ are insuffcient in themselves to prove the convergence of the continued fraction or of either sequence of alternate convergents. STIELTJEs, for his continued fraction, first demonstrated the convergence of the two sequences and then used the above relations to prove that their limits could be expressed as integrals of the form (3). This course can not be followed here since the two sequences are not always convergent, as will appear later.* Consequently in place of a general discussion we will take up certain cases wherein such supplementary conditions are imposed as to ensure simultaneously both the convergence of the sequence and the existence of the integral form for its limit.

* In deriving the integral (3) Stieltues employs also a certain accessory condition $\psi(a) \leqq \chi(b)$ (loc. cit., $\$ \S 41,43$ ). The proof of this condition assumes not only the convergence of the sequence considered but also a notable theorem concerning a LAURENT's power series (§§ 31-36). The latter can not always be applied to the continued fractions of this paper. 
The two most diverse suppositions which may be entertained are (1) that the minimum distance between two consecutive roots of $D_{n}$ as $n$ increases shall have a finite lower limit and (2) that the maximum distance between two consecutive roots in any arbitrary finite portion of the axis shall diminish indefinitely when $n$ is increased.

We shall take first the latter hypothesis and prove the convergence of the continued fraction at any assigned point $z$ exterior to the real axis. Consider first the even convergents. To prove their convergence it is necessary to prove that $\left|N_{2 n} / D_{2 n}-N_{2 n+2 m} / D_{2 n+2 m}\right|$ can be made less than any prescribed quantity $\epsilon$ by sufficiently increasing $n$. Now $N_{2 n} / D_{2 n}$ consists of the sum of $n$ terms $M_{i} /\left(z+z_{i}\right)$ while $N_{2 n+2 m} / D_{2 n+2 m}$ comprises a greater number of such terms. For comparison the latter may be divided into $n$ groups in the following manner. We know that between any two successive roots $z_{i}$ and $z_{i+1}$, of $D_{2 n}(-z)$, there is a root of $D_{2 n+2 m}(-z)$-call it $z_{(i)}^{(n+m)}$ —at which $\phi_{n+m}(u)$ receives an increment which first makes it equal to or greater than $\phi_{n}\left(z_{i}\right)$. This increment may be divided into two parts $M_{(i)}^{\prime}$ and $M_{(i)}^{\prime \prime}$, the former of which is an increment just sufficient to make $\phi_{n+m}(u)$ equal to $\phi_{n}\left(z_{i}\right)$. The first group of terms is to consist of

$$
\frac{M_{1}^{(n+m)}}{z+z_{1}^{(n+m)}}+\cdots+\frac{M_{(1)-1}^{(n+m)}}{z+z_{(1)-1}^{(n+m)}}+\frac{M_{(1)}^{\prime}}{z+z_{(1)}^{(n+m)}} \quad\left(M_{1}^{(m+n)}+\cdots+M_{(1)}^{\prime}=M_{1}\right),
$$

the second group of

$$
\frac{M_{(1)}^{\prime \prime}}{z+z_{(1)}^{(n+m)}}+\frac{M_{(1)+1}^{(n+m)}}{z+z_{(1)+1}^{(n+m)}}+\cdots+\frac{M_{(2)}^{\prime}}{z+z_{(2)}^{(n+m)}} \quad\left(\dot{M}_{(1)}^{\prime \prime}+\cdots+M_{(2)}^{\prime}=M_{2}\right),
$$

and so on. Then it is obvious that the difference between the $i$ th term of $N_{2 n} / D_{2 n}$ and the $i$ th group will have an absolute value not exceeding the larger of the two quantities

$$
M_{i}\left|\frac{1}{z+z_{i}}-\frac{1}{z+z_{i-1}}\right|=\frac{M_{i}\left|z_{i}-z_{i-1}\right|}{\left|z+z_{i}\right| \cdot\left|z+z_{i-1}\right|}, \quad M_{i}\left|\frac{1}{z+z_{i}}-\frac{1}{z+z_{i+1}}\right| .
$$

If $i=1, M_{i} / \mid 1\left(z+z_{1}\right)-1 /\left(z+z_{1}^{(n+m)} \mid\right.$ is to be substituted for the former of these expressions, and if $i=n$, a similar expression is to be substituted for the latter.

Take now any arbitrary segment of the real axis. The $n$ differences (27) can be divided into two sets comprising the differences which correspond respectively to roots of $D_{2 n}$ which lie within the segment and to those which lie without. Let $\delta_{i}$ be the greater of the distances between any root $z_{i}$ within the seg. ment and either adjacent root ${ }^{*}$ of $D_{2 n}$ (whether in or out of the segment), and let $p$ denote the distance of any point of the complex plane from the real axis.

\footnotetext{
* If $i=1$, we use the greater of the distances $\left|z_{1}-z_{1}^{(n+m)}\right|$ aud $\left|z_{1}-z_{2}\right|$.
} 
Then the larger of the differences (27) will be less than $M_{i} \delta_{i} / p^{2}$ and their sum less than $\sum M_{i} \delta_{i} / p^{2}$, the summation applying only to roots within the segment. But by virtue of our hypothesis the maximum of the distances $\delta_{i}$ may be indefinitely diminished by increasing $n$ sufficiently. Since also $\sum M_{i}$ is limited in value, it follows that we may make $\sum M_{i} \delta_{i} / p^{2}<\epsilon / 2$.

It remains to consider the second set of differences. By extending the segment sufficiently in both directions, $1 /\left|z+z_{i}\right|$ may be made as small as we choose for all roots which lie without the segment. Since also the distance between the first root without the segment and the first root within can be indefinitely diminished by increasing $n$, we can make $\left|1 /\left(z+z_{i}\right)-1 /\left(z+z_{i \pm 1}\right)\right|$ as small as is desired for each root $z_{i}$ without the segment, inclusive of the first root. Then since $\sum M_{i}$ is limited it follows that the sum of the second set of differences will also become less than $\epsilon / 2$, and consequently

$$
\left|N_{2 n} / D_{2 n}-N_{2 n+m} / D_{2 n+2 m}\right|<\epsilon / 2+\epsilon / 2=\epsilon .
$$

But this is what we had to prove.

In an exactly similar manner it can be shown that

$$
\left|N_{2 n} / D_{2 n}-N_{2 n+2 m+1} / D_{2 n+2 m+1}\right|<\epsilon .
$$

We conclude therefore that the continued fraction is convergent at any point not on the real axis. Furthermore, if any closed region $T$ is taken which lies entirely in the interior of the positive or the negative half plane, $p$ will have for this region a lower limit. The convergence is therefore uniform, and the limit of the continued fraction is accordingly holomorphic within $T$.

To put the limit into the form of an integral, we shall proceed now in the same manner as STIELTJES * and construct next a new function $\Phi(u)$ as follows. If $u$ is for the moment taken to be a fixed point of the real axis and $n$ assumes all values greater than a given integer, $\phi_{n}(u)$ will have both an upper and a lower limit. As the given integer is increased, the former limit can only decrease and the latter can only increase. Let $\psi(u)$ and $\chi(u)$ be the limits to which they approach, and put

$$
\Phi(u)=\frac{\chi(u)+\psi(u)}{2} .
$$

It is apparent that $\chi(u), \psi(u)$, and $\Phi(u)$ are all increasing functions of $u$ since $\phi_{n}(u)$ increases with $u$. If $c$ and $d$ be the upper and lower limits to the roots of $D_{2 n}(z)$, put

Then since

$$
F(z)=\int_{c}^{u} \frac{d \Phi(u)}{z+u} \quad(-\infty \leqq c<d \leqq+\infty) .
$$

* Loc. cit., $\S \S 41,44$. 


$$
\frac{N_{2 n}(z)}{D_{2 n}(z)}=\int_{+\infty}^{-\infty} \frac{d \phi_{n}(u)}{z+u}
$$

the function $F(z)$ bears exactly the same relation to $\Phi(u)$ as $N_{2 n}(z) / D_{2 n}(z)$ to $\phi_{n}(u)$. Moreover, as $\phi_{n+m}\left(z_{i}\right)-\phi_{n}\left(z_{i}\right)<0$, it follows that

$$
\Phi\left(z_{i}\right)-\phi_{n}\left(z_{i}\right) \leqq 0 \text {. }
$$

On the other hand, when $u=z_{i}-h$ ( $h$ infinitesimal and positive),

$$
\phi_{n+m}(u)-\phi_{n}(u)>0
$$

and hence $\Phi(u)-\phi_{n}(u) \geqq 0$. The difference $\Phi(u)-\phi_{n}(u)$ has accordingly properties similar to those of $\phi_{n+m}(u)-\phi_{n}(u)$ which we may use in the same manner to prove that we can make

$$
\left|F(z)-N_{2 n} / D_{2 n}\right|<\epsilon
$$

by increasing $n$ sufficiently; $F(z)$ is therefore the limit of the continued fraction.

13. Second special case.-We proceed now to the case in which the minimum distance between two consecutive roots of $D_{n}$ has a lower limit. By $\S 6$ the roots $1 / \bar{y}_{i}^{\prime}, 1 / \bar{y}_{i}^{\prime \prime}$ of $D_{2 n+1}(-z)$ move with increasing $n$ toward the origin of the z-plane. Call their limiting values $\xi_{i}^{\prime}, \xi_{i}^{\prime \prime}$. If any interval $(s,-s)$ of the real axis is taken, there will be a finite number of these values in the interval, and hence $D_{2 n+1}$ and $D_{2 n+2 m+1}$ for a sufficiently great value of $n$ will have precisely the same number of roots in the interval.

Consider those which lie upon the negative side of the origin. Since $1 / \bar{y}_{i}^{\prime \prime}<\xi_{i}^{\prime \prime}<1 / \bar{y}_{i-1}^{\prime \prime}$, we have $\psi_{n}\left(1 / \bar{y}_{i}^{\prime \prime}\right)=\psi_{n}\left(\xi_{i}^{\prime \prime}\right)$. Hence in the decomposition of $N_{2 n+1} / z D_{2 n+1}$ and $N_{2 n+2 n+1} / z D_{2 n+2+1}$ into partial fractions the terms which correspond to the negative roots of the denominators may be written as

$$
\frac{\psi_{n}\left(\xi_{i}^{\prime \prime}\right)-\psi_{n}\left(\xi_{i-1}^{\prime \prime}\right)}{z+1 / \bar{y}_{i}^{\prime \prime}}, \quad \frac{\psi_{n+m}\left(\xi_{i}^{\prime \prime}\right)-\psi_{n+m i}\left(\xi_{i-1}^{\prime \prime}\right)}{z+1 / y_{i}^{(n+m)}}
$$

Now $\psi_{n+m}\left(\xi_{i}^{\prime \prime}\right)=\psi_{n+m}\left(y_{i}^{(n+m)}\right)>\psi_{n}\left(\bar{y}_{i}^{\prime \prime}\right)=\psi_{n}\left(\xi_{i}^{\prime \prime}\right)$, which shows that $\psi_{n}\left(\xi_{i}^{\prime \prime}\right)$ increases with $n$ to a limit. The difference of the numerators of the two fractions (28) can therefore be made as small as desired. If $z$ is not on the real axis, this is also true of their denominators, since their roots have a common limit. The difference between the two fractions will therefore be diminished indefinitely by increasing $n$. A like result holds for the roots on the positive side of the origin and for the root at the origin. Hence the sum of the differences of the partial fractions for the roots of $D_{2 n+1}$ and $D_{2 n+2 n+1}$ within the interval can be made smaller than $\epsilon / 2$. 
By enlarging the interval chosen $1 /\left|z+1 / y_{i}\right|$ can be made arbitrarily small for all roots of the two polynomials which lie without the intervals. Hence the absolute values of the sums of the partial fractions

$$
\dot{\sum} M_{i} /\left|z+1 / y_{i}\right|, \quad \sum M_{i}^{(n+m)} /\left|z+1 / y_{i}^{(n+m)}\right|
$$

corresponding to these roots can be made less than $\epsilon / 4$. We have accordingly

$$
\left|\frac{N_{2 n+1}}{z D_{2 n+1}}-\frac{N_{2 n+2 m+1}}{z D_{2 n+2 m+1}}\right|<\frac{\epsilon}{4}+\frac{\epsilon}{4}+\frac{\epsilon}{2}=\epsilon,
$$

which shows that the sequence of odd convergents converges at any point $z$ not on the real axis.

The proof can be extended to all points on the real axis except $\xi_{i}^{\prime}, \xi_{i}^{\prime \prime}$, and it is easy to see that the convergence is uniform in any closed region which excludes these points.

The even convergents do not permit of similar treatment.

14. The methods of $\S \S 12,13$ can be extended to other cases, as for example the one mentioned in $\S 8$. We found there that the roots of $D_{n}$ had limiting positions separated by finite distances in the plane of $x$. In the z-plane there will be a point of condensation of these limiting positions at the origin. The principles of $\S 12$ can be applied to an arbitrarily small interval containing the origin as an interior point and the remainder of the axis can be treated as in $\S 13$.

\section{On the Convergence of the Continued Fraction and Corresponding Infinite Series.}

15. Character of the series. - It is well known that to every continued fraction of the form (4) there belongs an infinite series,

$$
c_{0}-c_{1} x+c_{2} x^{2}-c_{3} x^{3}+\cdots
$$

with which the expansion of $N_{n}(x) / D_{n}(x)$ in ascending powers of $x$ agrees for a number of terms equal to the sum of the degrees of numerator and denom inator, increased by 1 . Not only can the series be obtained from the continued fraction, but conversely, if the series is given, the continued fraction can be found. If, in short, we put*

$$
A_{n}=\left|\begin{array}{cccc}
c_{0} & c_{1} & \cdots & c_{n-1} \\
c_{1} & c_{2} & \cdots & c_{n} \\
\cdot & \cdot & \cdot & \cdot \\
c_{n-1} & c_{n} & \cdots & c_{2 n-2}
\end{array}\right|, \quad B_{n}=\left|\begin{array}{cccc}
c_{1} & c_{2} & \cdots & c_{n} \\
c_{2} & c_{3} & \cdots & c_{n+1} \\
\cdot & \cdot & \cdot & \cdot \\
c_{n} & c_{n+1} & \cdots & c_{2 n-1}
\end{array}\right|, A_{0}=B_{0}=1,
$$

* Stieltues, loc. cit., $\& 11$. The expressions for the elements of the continued fraction in terms of those of the series go back at least to Froberius, Crelle's Journal, vol. 90 (1881), pp. 1-5. 
and also

$$
a_{2 n}=\frac{A_{n}^{2}}{B_{n-1} B_{n}}, \quad a_{2 n+1}=\frac{B_{n}^{2}}{A_{n} A_{n+1}},
$$

we have then for the coefficients of the continued fraction

$$
b_{n}=\frac{1}{a_{n-1} a_{n}} .
$$

To furnish therefore a continued fraction in which $b_{2 n}$ and $b_{2 n+1}$ have like signs, the series (29) must be subjected to the restrictions

$$
A_{n}>0, \quad B_{n} \neq 0 .
$$

On the other hand, if $b_{2 n-1}$ and $b_{2 n}$ are to be of like sign, we have

$$
B_{n}>0, \quad A_{n} \neq 0 .
$$

The quantities $a_{n}$ are themselves the coefficients of a continued fraction of the form (1), to which Stieltues gives the preference. To the conditions (30) and (31) correspond two classes of continued fractions in which $a_{2 n+1}$ and $a_{2 n}$ respectively are positive.

The awkward secondary conditions $B_{n} \neq 0, A_{n} \neq 0$ were necessary above to prevent $b_{n}$ from becoming infinite and the continued fraction in consequence illusory. By reference to (8) it will be seen at once that the even convergents of (4) generate by themselves a continued fraction which, in terms of $z$, has the familiar form

in which

$$
z\left[\frac{\lambda_{0}}{z+d_{1}}-\frac{\lambda_{1}}{z+d_{2}}-\frac{\lambda_{2}}{z+d_{3}}-\cdots\right]
$$

$$
\begin{gathered}
\lambda_{0}=b_{0}=1, \quad \lambda_{n}=b_{2 n} b_{2 n+1}=\frac{A_{n-1} A_{n+1}}{A_{n}^{2}}, \\
d_{1}=b_{2}=\frac{B_{0} B_{1}}{A_{1}^{2}}, \quad d_{n+1}=b_{2 n+1}+b_{2 n+2}=\frac{A_{n+1}^{2} B_{n-1}+A_{n}^{2} B_{n+1}}{A_{n} A_{n+1} B_{n}} .
\end{gathered}
$$

Here the $\lambda_{n}$ depend only upon the $A_{n}$, and the $d_{n}$ can not become infinite as long as $A_{n} \neq 0$, since $B_{n}$, which appears in the denominator of $d_{n+1}$, is contained also as a factor in its numerator.* The continued fraction (32) therefore can not become illusory if $A_{n} \neq 0$ for all values of $n$.

* For verification of this statement it is convenient to refer forward to 820 . In the notation of this paragraph $A_{n} \equiv c_{n-1, n}$ or $B_{n}=c_{n, n}$. Now by a familiar theorem of determinants

Hence

$$
\begin{gathered}
c_{\alpha \beta}^{2}=c_{\alpha+1, \beta} c_{\alpha-1, \beta}-c_{\alpha, \beta+1} c_{\alpha, \beta-1} . \\
A_{n}^{2}=B_{n} c_{n-2, n}-c_{n-1, n+1} B_{n-1} \\
A_{n+1}^{2}=B_{n+1} c_{n-1, n+1}-c_{n, n+2} B_{n} .
\end{gathered}
$$

The substitution of these values in the numerator of $d_{n+1}$ gives the desired result. 
If $B_{n} \neq 0$, we obtain in similar manner from the odd convergents a continued fraction

in which

$$
1-\frac{\lambda_{1}^{\prime}}{z+\bar{d}_{1}^{\prime}}-\frac{\lambda_{2}^{\prime}}{z+\bar{d}_{2}^{\prime}}-\cdots
$$

$$
\lambda_{1}^{\prime}=b_{2}, \quad \lambda_{n}^{\prime}=b_{2 n-1} b_{2 n}=\frac{B_{n-2} B_{n}}{B_{n-1}^{2}}, \quad d_{n}^{\prime}=b_{2 n}+b_{2 n+1} .
$$

We conclude therefore that the secondary conditions in (30) and (31) can be dispensed with if in the one case only the even convergents of (4) are considered and in the other only the odd convergents.

16. On the radius of convergence of the series.-It is clear that the series which satisfy (30) and (31) respectively permit of similar treatment. We shall confine ourselves here to those which satisfy the former. condition.

The necessary and sufficient conditions for convergence can then be obtained as follows. It is known that when $A_{n}>0$ for all values of $n$, any minor formed symmetrically from $A_{n}$ by deleting corresponding rows and columns must also be positive. ${ }^{*}$ Hence $c_{2 n}>0$ and

$$
\left|\begin{array}{ll}
c_{2 n} & c_{2 n+2} \\
c_{2 n+2} & c_{2 n+4}
\end{array}\right|>0, \quad \text { i. e., } \quad \frac{c_{2 n+4}}{c_{2 n+2}}>\frac{c_{2 n+2}}{c_{2 n}} .
$$

If, therefore, $c_{2 n+2} / c_{2 n}$ increases without limit, the series is divergent. On the other hand, if it increases to a limit $L^{2}$, it can be shown that the radius of convergence of $(29)$ is equal to $1 / L$. This is true obviously after the odd powers are omitted from the series. But since

$$
\left|\begin{array}{ll}
c_{2 n} & c_{2 n+1} \\
c_{2 n}+1 & c_{2 n+2}
\end{array}\right|>0
$$

$\left|c_{2 n+1}\right|$ must be less than one or both of the coefficients $c_{2 n}$ and $c_{2 n+2}$. The odd powers have then a circle of convergence as great as the even powers, and hence the radius for the entire series must be equal to $1 / L$.

Since the first $2 i$ terms of the series can be obtained by the expansion of $N_{2 n} / D_{2 n}$ for sufficiently large values of $n$, we have from (19)

$$
\frac{c_{2 i}}{c_{2 i-2}}=\frac{\sum_{s=1}^{n} M_{s} z_{s}^{2 i}}{\sum_{s=1}^{n} M_{s} z_{s}^{2 i-2}}<\bar{z}^{2},
$$

*Sylvester, Philosophical Magazine, ser. 4, vol. 4 (1852), pp. 140-141. 
in which $\bar{z}$ denotes the greatest absolute value of any root of $D_{2 n}(z)$. It follows that $L$ is equal to or less than the limiting value of $\bar{z}$. This proves that the radius of convergence is at least as great as the reciprocal of the limit of the absolute value of the numerically largest root of $D_{2 n}(z)$ or, in other words, as great as the limiting distance from the $x$-origin to the nearest root of $D_{2 n}(x=1 / z)$.

17. The region of convergence for the continued fraction.-The question of the convergence of the continued fraction, unlike that of the series, appears to be a very complicated one. The form of the region of convergence, however, can be almost completely determined. First, it should be shown that the continued fraction converges uniformly over some closed finite region.* The region can then be extended by means of a notable theorem discovered by StiEltJEs. $\dagger$ Let, namely, $f_{1}(z), f_{2}(z), \ldots$ denote an infinite sequence of functions which are holomorphic within a closed area $S$, then if $\sum f_{n}(z)$ is uniformly convergent in some region $S_{2}$ lying within $S$ and if, furthermore, $f_{1}(z)+f_{2}(z)+\cdots+f_{n}(z)$ has an upper limit independent of $n$ in any region $S_{1}$ which includes $S_{2}$ but is contained in the interior of $S$, the series will also converge uniformly in $S_{1}$, and its limit will be holomorphic in the interior of $S$. For application we will put $f_{1}(z)+f_{2}(z)+\cdots+f_{n}(z)$ equal to the $n$th convergent (or to the $n$th term of any particular sequence which we may choose to consider). From equations (19) to (21) it is evident that $\left|N_{n}(z) / D_{n}(z)\right|$ will have an upper limit independent of $n$ in any regions lying wholly at a finite distance from the real axis. The region of convergence can therefore be extended so as to cover the interior of a half plane. If, furthermore, there is any segment of the real axis which is free from roots of $D_{n}$ (or roots of the denominator of the $n$th term of the sequence chosen), the region can be extended over this segment so as to include also the interior of the other half plane.

18. The common cases of convergence.-The continued fractions of most frequent occurrence are those in which $\left|b_{n}\right|$ has an upper limit $k$. These I have previously considered, $\ddagger$ and have shown that the continued fraction (4) converges within at least a circle of radius $1 / 4 k$, having its center in the origin. The convergence is uniform within any interior circle, and the principle of STIELTJEs stated in $\$ 17$ extends the region of convergence over the entire interior of the positive and negative half planes.

* This is, for example, true of our continued fraction if

$$
\frac{1}{D_{n} D_{n+1}}-\frac{b_{n+2} x}{D_{n+1} D_{n+2}}+\frac{b_{n+2} b_{n+3} x^{2}}{D_{n+2} D_{n+3}} \cdots
$$

is absolutely convergent at any one point not on the real axis.

† Loc. cit., $\$ 30$. For an extension of this theorem by OsGood see the Annals of Mathematics, ser. 2 , vol. 3 , p. 133.

$\ddagger$ Loc. cit., p. 477. 
A second interesting case is that in which the absolute values of the negative (positive) coefficients of (4) but not the positive (negative) coefficients have an upper limit. The continued fraction can then be reduced to a dependence upon that of StieltJes, but for this purpose the even and odd convergents must be treated separately. If $b_{2 n} b_{2 n+1}>0$, take the even coefficients which generate the continued fraction (32), and suppose that the absolute values of the negative $b_{n}$ have an upper limit less than $C$. The coefficients $d_{n+1}$ can be made positive by the substitution of $z^{\prime}+2 C$ for $z$. Let the new values be denoted by $d_{n+1}^{\prime}$. Then the values of the $b_{n}$ to correspond will be found from the equations :

$$
\lambda_{n}=b_{2 n}^{\prime} b_{2 n+1}^{\prime}, \quad b_{2}^{\prime}=b_{2}+2 C, \quad d_{n+1}^{\prime}=d_{n+1}+2 C=b_{2 n+1}^{\prime}+b_{2 n+2}^{\prime} .
$$

Since the value of $\lambda_{n}$ remains unaltered in the transformation while $d_{n}^{\prime} \geqq d_{n}$, it follows by easy induction from the last equations that

$$
b_{2 n}^{\prime}>\left|b_{2 n}\right|, \quad 0<b_{2 n+1}^{\prime}<\left|b_{2 n+1}\right| \text {. }
$$

The even convergents of (18) are therefore also the even convergents of another continued fraction of like form,

in which $b_{n}^{\prime}>0$.

$$
\frac{1}{z^{\prime}}+\frac{b_{2}^{\prime}}{1}+\underline{z_{3}^{\prime}}+\frac{b_{4}^{\prime}}{1}+\cdots,
$$

It is obvious that the convergence or divergence of any sequence of convergents is not affected by our transformation, for the latter is merely a change of origin. Now (34) is only a slightly altered form of the continued fraction of StIELTJEs, the alternate convergents of which were shown by him to converge over the entire plane of $z^{\prime}$ with the exception of the whole or a part of the negative half of the real axis. Our sequence of even convergents was therefore convergent over the plane of $z$ with the exception of the portion of the real axis to the left of the point $z=2 C$.

It should not, however, be hastily assumed that the same is true of the odd convergents. Although the continued fractions (32) and (33) had their origin in a single continued fraction (4), they will not correspond after the transformation to the same continued fraction (34), and thus the two sets of convergents lose their initial property of making together a single continued fraction of the form (4).

This fact can be inferred most easily from the equation :

$$
D_{2 n-2}(z) N_{2 n-1}(z)-z D_{2 n-1}(z) N_{2 n-2}(z)=\lambda_{0} \lambda_{1} \cdots \lambda_{n-1} .
$$

This relation uniquely determines $N_{2 n-1}, D_{2 n-1}$ when the continued fraction (32) satisfied by the even convergents is known. When, now, the substitution 
$z=z^{\prime}+2 C$ is made in (32), a new pair of polynomials must be chosen after the transformation which satisfy the equation

$$
D_{2 n-2} N_{2 n-1}^{\prime}-z^{\prime} D_{2 n-1}^{\prime} N_{2 n-2}=\lambda_{0} \lambda_{1} \cdots \lambda_{n-1} .
$$

The new pair is evidently connected with the old by the equations

$$
N_{2 n-1}^{\prime}=N_{2 n-1}+t_{n} N_{2 n-2}, \quad z^{\prime} D_{2 n-1}^{\prime}=z D_{2 n-1}+t_{n} D_{2 n-2},
$$

in which $t_{n}$ is a constant which must be so chosen that the absolute term shall disappear in $z D_{2 n-1}+t_{n} D_{2 n-2}$, when expressed in terms of $z^{\prime}$.

The new rational fractions $N_{2 n-1}^{\prime} / z^{\prime} D_{2 n-1}^{\prime}$ are the odd convergents of the continued fraction of StIELTJEs given in (34) and form in consequence a convergent sequence. The equation

$$
\frac{N_{2 n-1}}{z D_{2 n-1}}=\frac{N_{2 n-1}^{\prime}-t_{n} N_{2 n-2}}{z^{\prime} D_{2 n-1}^{\prime}-t_{n} D_{2 n-2}}
$$

now makes it plain that when (34) is convergent, $N_{2 n-1} / z D_{2 n-1}$ is, in general, also convergent, but an exceptional case is evidently possible.* No definite conclusion, then, is reached concerning the sequence of the odd convergents of (4) by means of the transformation.

When $B_{n}>0$, the rôle of the odd and even convergents is interchanged.

19. The less common cases.-The cases in which neither the positive nor the negative $b_{n}$ have numerically an upper limit offer greater difficulty. Two interesting cases of this description can be derived by transformation from the continued fraction of Stieltues. For this purpose place $z=-w^{2}$ in (1). By a simple reduction it can be brought into the form

$$
-\frac{1}{w}\left[\frac{1}{w}-\frac{b_{2}}{w}-\frac{b_{3}}{w}-\cdots\right] \quad\left(b_{n}=\frac{1}{a_{n} a_{n-1}}\right) .
$$

We can then regard it as a special case of (32) in which $d_{n}=0$. Hence its convergents are the even convergents of another continued fraction (34) in which $b_{2 n}^{\prime} b_{2 n+1}^{\prime}=\lambda_{n}=b_{n+1}$ and $b_{2 n+2}^{\prime}=-b_{2 n+1}^{\prime}+C$, if $C$ is properly chosen.

The two cases distinguished by STIELTJES must now be considered separately. When $\sum a_{n}$ is convergent, $\lim \left(1 / a_{n} a_{n+1}\right)=\lim b_{2 n}^{\prime} b_{2 n+1}^{\prime}=\infty$. Since also $b_{2 n+2}^{\prime}=-b_{2 n+1}^{\prime}+C$, there can be no limit numerically to the positive and nega-

* This is in agreement with the fact that in discussing the "problem of moments" STIELTJES finds that the continued fraction for

may be divergent while that for

$$
\int_{0}^{\infty} \frac{f(z+\lambda)}{z+u+\lambda} d z
$$

is convergent ; loc. cit., $\S 78$.

$$
\int_{0}^{\infty} \frac{f(z) d z}{z+u}
$$


tive coefficients in (34), and it is therefore such a continued fraction as we wished to consider. Now Stieltues has shown that when $\sum a_{n}$ is convergent in his continued fraction, its even and odd convergents have distinct limits, and these limits expressed in terms of $z$ are meromorphic functions of genre 0 . In correspondence with this the even convergence of (34) split up into two sequences, each sequence having a limit which is meromorphic in the plane of $w=z^{\prime}+C$ and is of genre 1 or 0 . In regard to the odd convergents of (34), we have seen in $\S 6$ that the roots of $D_{2 n+1}$ with an increase of $n$ move toward the origin of the plane of $w=1 / x$. Since, moreover, these roots are separated by those of $D_{2 n+2}$ which for alternate values of $n$ approach as their limiting positions the poles of two meromorphic functions, the distance between two consecutive roots of $D_{2 n+1}$ in any segment of the axis must have a finite lower limit, however much $n$ be increased. The conditions of $\S 13$ therefore are fulfilled. Hence the odd convergents furnish only a single limiting function, and it is easy to infer that this function is meromorphic and of genre 1 or 0.

When $\sum a_{n}$ is divergent, the continued fraction of S'TIELTJES is convergent, and hence also the even convergents of (34), if derived in the manner just indicated. The latter continued fraction, however, is not necessarily one in which the absolute values of the coefficients have no upper limit. To secure a case of this sort we shall so restrict the $a_{n}$ that the analytic function which is the limit of (1) can not be continued analytically across any portion of the negative half of the real axis. Stieltues has shown that this is possible. $\dagger$ By the transformation $z=-w^{2}$ the half axis is replaced by the entire axis, and the latter is therefore a natural boundary for the limits of the even convergents of (34). Now by $\S 18$ this is impossible if $\left|b_{n}\right|$ is limited. Since, furthermore, $b_{2 n+1}^{\prime}=-b_{2 n+2}^{\prime}+C$, neither the positive elements nor the absolute values of the negative elements will have an upper limit. We conclude then that the continued fraction (34) falls into the category to be considered.

Each point of the axis of $w \equiv z^{\prime}+C$ must be a point of condensation of the roots of the $2 n$th convergent of $(34),(n=1,2, \ldots)$. For were this not so, the limit of this convergent by $\S 17$ could be continued analytically across the axis. It follows then by $\S 12$ that (34) is convergent. The corresponding series $P\left(1 / z^{\prime}\right)$ is divergent; for if it were convergent we could pass by means of the transformation

$$
-\left(z^{\prime}+C\right)^{2}=-w^{2}=z
$$

† Loc. cit., \&59. This is a consequeuce of his equation

$$
\operatorname{Lim}_{\epsilon=0}[\Phi(-x+\varepsilon i)-\Phi(-x-\varepsilon i)]=\pi i[\phi(\bar{x})+\phi(\stackrel{+}{x})],
$$

which gives the difference of the limiting values of the continued fraction when a point $-x$ of the negative axis is approached from opposite sides. If $\phi(x)$, the integrand of $(3)$, is nonanalytic, so is the integral $\Phi(x)$. 
to the series (2) which corresponds to the continued fraction of Stieltues, and this series would be convergent. But the series (2) was shown by STIELTJES to be divergent, when his continued fraction represents a function incapable of analytic continuation across any portion of the real axis. We arrive thus at the interesting result that a convergent continued fraction (34) and the corresponding divergent series may define in the positive and negative half planes two distinct analytic functions having the real axis as a common natural boundary.

It should be noted that the two analytic functions take conjugate imaginary values in two points situated symmetrically with respect to the axis. Our continued fraction thus offers a method of continuing the analytic function across the natural boundary. The character of this continuation seems to be in essential accord with the ideas of BOREL and FABRY for such a continuation.

\section{On the Table of Approximants for the Series.}

20. The table of PADE. - In the previous sections it has been found convenient to consider separately the even and the odd convergents. The essential reason for this will appear if a table of approximants for a series

$$
c_{0}+c_{1} x+c_{2} x^{2}+\cdots *
$$

is formed after the manner of PADE. $\dagger$

Among all the rational functions in which the degrees of the numerators and denominators do not exceed two prescribed integers, $p$ and $q$ respectively, there is one rational function and, taken in its lowest terms only one, whose expansion in a series of ascending powers of $x$ agrees with (36) for as great a number of successive terms as possible. Such a fraction I shall term an approximant and denote by $U_{p q} / V_{p q}$. PADE arranges all the approximants in a table of double entry :

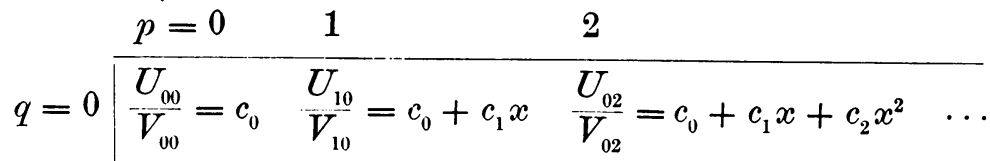

$$
\begin{aligned}
& q=1 \mid \begin{array}{ccc}
U_{01} & U_{11} & U_{21} \\
\hdashline V_{01} & V_{11} & V_{21} \\
U_{012} & \frac{U_{12}}{V_{12}} & U_{22} \\
\frac{V_{02}}{V_{22}} & &
\end{array}
\end{aligned}
$$

* The reader will note that the sign of $c_{2 i-1}$ has been changed. This has been done to facilitate comparison with the work of FROBENIUS hereafter.

† Thesis: Sur ln représentation approchée d'une fonction par des fractions rationnelles, A $\mathbf{n} \mathbf{n a l}$ es de l'Ecole Normale, sér. III, vol. 9 (1892), supplément.

Trans. Am. Math. Soc. 22 
Generally the series for $U_{p q} / V_{p q}$ agrees with (36) for its first $p+q+1$ terms. This is always the case when the fraction, taken in its lowest terms, appears but once in the table. When no rational fraction occurs more than once in the table, the table is said by PADE to be normal. The necessary and sufficient condition for the existence of such a table is that none of the determinants

shall vanish.*

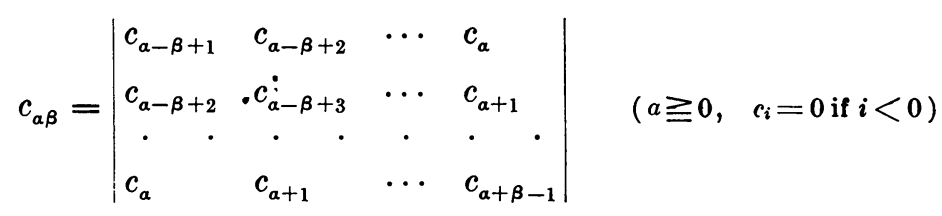

Obviously all the approximants which lie upon a line perpendicular to the principal diagonal of the table belong to the same value of $p+q+1$. If, then, the table is normal, they each give the same number of successive terms in (36). Hence they may be said to approximate to the series in equal degree or to be "equally advanced" in the table.

When the table is not normal, all approximants which are equal to one another fill up a square whose sides are parallel to those of the table. If any such square contains $n^{2}$ terms, it may be called an irregularity of the $(n-1)$ th order.

Each approximant in the table is surrounded by eight others, unless it lies upon the border when it is enclosed by five. These are called the contiguous approximants.

21. The regular continued fractions.-Consider now any normal table, and let any succession of approximants be taken, beginning with a term on the border and passing always from one approximant to another which is contiguous to it but more advanced in the table. PADE shows that such a sequence of approximants are the successive convergents of a continued fraction, all of whose elements are polynomials in $x$. This holds also for a non-normal table, provided that in every succession of equal approximants which occur in the sequence all but the first are erased.

The continued fraction is called regular when its partial numerators are all of the same degree and likewise its denominators, certain specified and simple irregularities being permitted in the first one or two partial fractions. In a normal table a regular fraction is obtained $\dagger$ when the approximants lie

(1) upon a horizontal or vertical line;

(2) upon a straight line parallel to or coincident with the principal diagonal ;

* PADÉ, loc. cit., § 31.

† Loc. cit., \& 53 . 
(3) upon a step-like line, proceeding alternately one term horizontally to the right and one vertically downwards.

Only the second and third types of continued fractions concern us here. If these begin with the $p$ th approximant of the horizontal border, they have the form

$$
\begin{gathered}
c_{0}+c_{1} x+\cdots+c_{p-1} x^{p-1}+\left(\frac{c^{p} x^{p}}{1+l_{1} x}+\frac{k_{2} x^{2}}{1+l_{2} x}+\frac{k_{3} x^{2}}{1+l^{3} x}+\cdots\right) \\
c_{0}+c_{1} x+\cdots+c_{p-1} x^{p-1}+\left(\frac{c_{p} x^{p}}{1}+b_{2} x-\frac{b_{3} x}{1}+\cdots\right)
\end{gathered}
$$

if with the $p+1$ th approximant of the vertical border,

$$
\begin{gathered}
\frac{U_{0, p}}{V_{0 p}}+\frac{k_{2} x^{p+1}}{1+l_{2} x}+\frac{k_{3} x^{2}}{1+l_{3} x}+\cdots, \\
\frac{U_{0 p}}{V_{0 p}}+\frac{b_{2} x^{p+1}}{1}+\frac{b_{3} x}{1}+\cdots
\end{gathered}
$$

To determine the coefficients of these continued fractions it will be convenient to take $c_{0}=1$ and then to put the constant terms of $U_{p q}$ and $V_{p q}$ equal to 1. The numerators and denominators of the convergents thereby are made identical with certain pairs of polynomials, $U_{p q}$ and $V_{p q}$, so selected that each pair is followed by another more advanced but contiguous to it. Hence the coefficients in any partial fraction will be the coefficients of some relation connecting three consecutive polynomials $V_{p q}$. The relations of this character have been determined by Frobenius * and run as follows:

$$
\begin{gathered}
V_{a+1, \beta}=V_{a \beta}-\frac{c_{a, \beta-1} c_{a+1, \beta+1}}{c_{a, \beta} c_{a+1, \beta}} x V_{a, \beta-1}, \\
V_{a+1, \beta+1}=V_{a+1, \beta}-\frac{c_{a \beta} c_{a+2, \beta+1}}{c_{a+1, \beta} c_{a+1, \beta+1}} x V_{a \beta}, \\
V_{a+1, \beta+1}=\left[1+\left(\frac{c_{a+1, \beta+2} c_{a, \beta-1}-c_{a+2, \beta+1} c_{a-1, \beta}}{c_{a \beta} c_{a+1, \beta+1}}\right) x\right] V_{a \beta} \\
-\frac{c_{a-1, \beta-1} c_{a+1, \beta+1}}{c_{a \beta}^{2}} x^{2} V_{a-1, \beta-1} .
\end{gathered}
$$

* Crelle's Journal, vol. 90 (1881), p. 1, equations (4), (5), and (8) of $\S 2$. These equations have been appropriately modified so as to make the coefficient of $V_{a \beta}$ equal to 1 . When taken with the analogons equations for the $U_{a \beta}$, they give the continued fractions of PADÉ for a normal table. PADÉ, however, was the first to note the advantage and significance of the arrangement of the approximants into a table of double entry, and the importance of the various questions which attach themselves to such a table. 
Equations (37) and (38) serve to determine the values of the $b_{n}$ and equation (39) the values of the $k_{n}$ and $l_{n}$.

A comparison of our fundamental continued fraction (4) with (3") shows that it is of the third type. Since also it is regular from the very outset, it starts from the corner of the table, and the line of approximants which form its convergents proceeds downward in the following manner :

$$
\begin{array}{lll}
\frac{U_{00}}{V_{00}} & & \\
\frac{U_{01}}{V_{01}} & \frac{U_{11}}{V_{11}} & \\
& \frac{U_{12}}{V_{12}} & \frac{U_{22}}{V_{22}} \\
& & \\
& & \frac{U_{23}}{V_{23}} \cdots
\end{array}
$$

The odd convergents fill the principal diagonal of the table and the even convergents a parallel file, and hence they form separately two continued fractions of the second type. In the case considered by Stieltues the two files were shown to converge to distinct limits when $\sum a_{n}$ is convergent.* Thus his work, when put into relation with PADE's table, brings to light the important fact that the table may give rise to continued fractions which represent different functions. $\dagger$ The extension of his work found in the present paper shows also that it is possible for one file to converge and not the other, or even for different sets of convergents chosen from the same file to converge to different limits.

22. Character of the table when $A_{n}>0$. - It has been already noted that our continued fraction (4) defines uniquely a series and therefore a table of approximants. We proceed now to examine the character of the table when $A_{n}>0$. For this purpose it will be necessary to fix the signs of the determinants $c_{\alpha \beta}$ for which $\alpha-\beta$ is an odd integer.

If $\alpha-\beta$ is odd and positive or is equal to -1 , the determinant $c_{\alpha \beta}$ is a minor of $A_{n}$ formed symmetrically from its rows and columns, and hence by the theorem of SyLvester previously cited must be positive.

Suppose next that $\alpha-\beta=-3$. From a familiar theorem for the product of a determinant by one of its minors we obtain

* In the case of the exponential function PADÉ has shown that the different continued frac. tions lead to a common limit, Annales de l'Ecole Normale, ser. III, vol. 16 (1899).

† The bearing of the work of STIELTJ Es has been pointed out previously by PADÉ ; Com ptes Rend us, vol. 132 (1901), p. 912. No further examination of the table is made as in the present paper. 


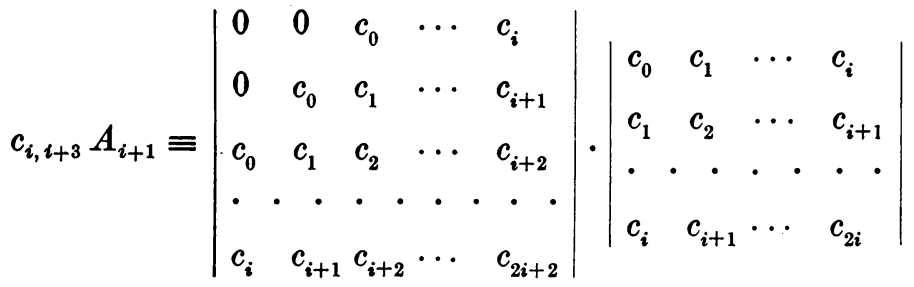

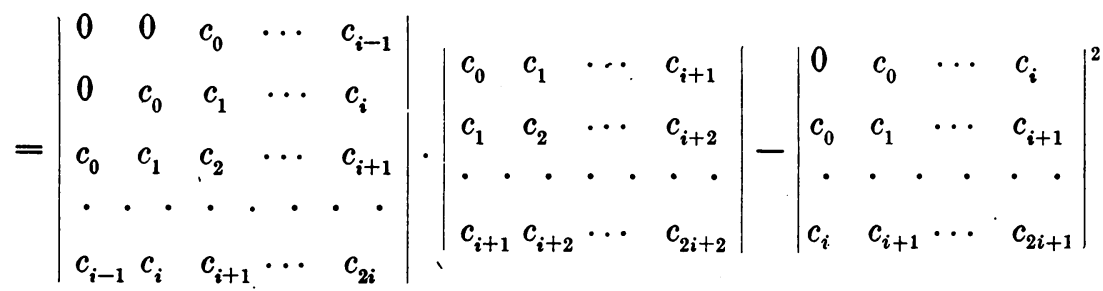

$$
\begin{aligned}
& \equiv c_{i-1, i+2} A_{i+2}-()^{2} \text {. }
\end{aligned}
$$

If therefore $c_{i-1, i+2}$ is negative, $c_{i, i+3} A_{i+1}$ and hence $c_{i, i+3}$ must also be negative. But $c_{0,3}=\left(-c_{0}\right)^{3}$. Since this is negative, $c_{1,4}$ must be negative, then $c_{2,5}$, and so on. In other words, $c_{i, i+3}$ is negative for all values of $i$.

It thus appears that the sign of $c_{i, i+2 n+1}$ is independent of $i$ when $n=1$ and is $(-1)^{n}$. We will now show that if this is true for all values of $n$ from 1 to $m$ inclusive, it must be true for $n=n \iota+1$ and hence universally true. For proof we shall use the same theorem as before for the product of a determinant and its minor. This gives in general

$$
c_{i, i+2 m+3} c_{i, i+2 m+1}=c_{i-1,(i-1)+2 m+3} c_{i+1,(i+1)+2 m+1}-\left(c_{i, i+2 m+2}\right)^{2} .
$$

If, therefore, $c_{i-1,(i-1)+2 m+3}$ and $c_{i+1,(i+1)+2 m+1}$ have opposite signs, $c_{i, i+2 m+3}$ and $c_{i, i+2 m+1}$ have also opposite signs. But the second and fourth of these quantities have the sign $(-1)^{m}$ by hypothesis. Hence if $c_{i-1,(i-1)+2 m+3}$ for any value of $i$ has the sign $(-1)^{m+1}$, the value of $i$ can be increased by successive units without destroying the property. But for the smallest value of $i$ we have

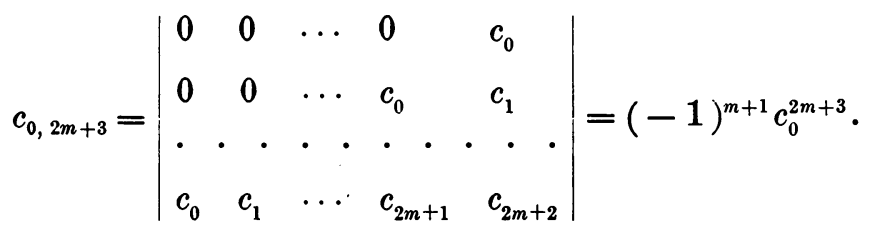

This completes the proof that $c_{i, i+2 n+1}$ has the $\operatorname{sign}(-1)^{n}$ for all values of $i$ and $n$.

When $B_{n}>0$, it can be shown in similar manner that $c_{a, \beta}$ is positive if $\alpha-\beta$ is an even positive integer or 0 , and has the sign $(-1)^{n}$ if $\alpha-\beta=-2 n$. 
When therefore the two conditions $A_{n}>0, B_{n}>0$ hold simultaneously, $c_{\alpha \beta}$ can not vanish for any value of $\alpha-\beta$, positive or negative. We thus arrive at the conclusion:

Theorem VIII.-The table of approximants for the series of STIELTJES is a normal one.

When the condition $B_{n}$ is dropped, the normal character of the table is not always conserved. For one or more even values of $\alpha-\beta$ the determinant $c_{\alpha \beta}$ may vanish, and in consequence some of the continued fractions furnished by the table may become illusory. It will be noticed, however, that in (39) the difference of the subscripts, $\alpha-\beta$, is the same not only for the three polynomials $V_{p q}$ which there appear but also for the $c_{\alpha \beta}$ occurring in the denominators of the coefficients. The equation therefore has a significance for any odd value of $\alpha-\beta$, and hence the alternate diagonal files of the table for which $\alpha-\beta$ is odd furnish continued fractions of the second type. In these continued fractions it is apparent that no two consecutive convergents can be identical. Therefore the irregularities which appear in the table, being in the form of identical approximants, can contain only $2^{2}$ elements. In other words :

Theorem IX.-If $A_{n}>0$ (or $B_{n}>0$ ), all irregularities in the table must be of the first order.

23. The zeros and infinities of the approximants.-We proceed now to the study of the continued fractions furnished by the alternate diagonal files of the table. These have the form $\left(2^{\prime}\right)$ or $\left(2^{\prime \prime}\right)$, the values of the coefficients being obtained from (39). For example, the $(2 p-1)$ th diagonal file to the right of the principal diagonal gives

$$
c_{0}+c_{1} x+c_{2} x^{2}+\cdots+c_{2 p-1} x^{2 p-1}+\frac{c_{2 p} x^{2 p}}{1+l_{2} x}+\frac{k_{2} x^{2}}{1+l_{3} x}+\cdots
$$

in which

$$
k_{n}=-\frac{c_{\alpha-1, \beta-1} c_{\alpha+1, \beta+1}}{c_{\alpha \beta}^{2}} \quad(a=n+2 p-1, \beta=n) .
$$

Now we have seen that when $i-j$ is odd, $c_{i j}$ has a sign depending only upon the subscript difference. Therefore $k_{n}$ is negative, the relation (39) is analogous to (8), and the continued fraction (40), after the omission of the irregularity at the outset, is precisely such a continued fraction as was satisfied by the even convergents of (4). Our conclusions regarding these convergents were based upon the relation (8) and not upon the form of (4). Accordingly we may express $n$th convergents of $(40)$ as

$$
\frac{N_{n}^{\prime}}{D_{n}^{\prime}}=c_{n}+\cdots+c_{2 p-1} x^{2 p-1}+\frac{c_{2 p} x^{2 p} N_{2 n}}{D_{2 n}}
$$


in which $N_{2 n} / D_{2 n}$ denotes a fraction having the same properties as the $2 n$th convergent of (4).

The roots of $D_{n}^{\prime} \equiv D_{2 n}$, as we have previously seen, are real. To ascertain the number of real roots of $N_{n}^{\prime}$ it will be necessary to know first the number of such roots of

$$
c_{0}+c_{1} x+\cdots+c_{2 p-1} x^{2 p-1}=0 .
$$

Elsewhere* I have shown that only one root of this equation is real, and this was done by proving that

$$
\frac{c_{0}+c_{1} x+\cdots+c_{2 p-1} x^{2 p-1}}{x^{2 p-1}}
$$

is an increasing function of $z=1 / x$ and therefore a decreasing function of $x$. Now $x N_{2 n} / D_{2 n}$ is either always an increasing or always a decreasing function of $x$ because the roots of numerator and denominator separate each other. But the fraction is zero at the origin and positive for small positive values of $x$. It must then be an increasing function. Hence as $x$ traverses the real axis, starting from the origin and returning finally to it from the opposite side, $x N_{2 n} / D_{2 n}$ will become equal to the decreasing function (41) once and but once between two consecutive roots of $D_{2 n}$ and once between the origin and each adjoining root. Consequently the number of real roots of $N_{n}^{\prime}$ is exactly $n+1$, and $2 p-2$ roots will be imaginary. The real roots alternate with the roots of $D_{n}^{\prime}$ on each side of the origin, and the root closest to the origin on either side is a root of $N_{n}^{\prime}$.

We turn now to the approximants on the $(2 p-1)$ th diagonal file below the principal diagonal. It is easy to see that the reciprocals of the approximants are themselves approximants to the expansion (formally) of

$$
\frac{1}{\left(c_{0}+c_{1} x+c_{2} x^{2}+\cdots\right)}
$$

in ascending powers of $x$. The latter can be obtained to any desired number of terms by expanding $V_{n+1, n} / U_{n+1, n}$ into series for sufficiently large values of $n$. But this fraction, in accordance with the preceding paragraph, has only real zeros and infinities which are situated relatively to one another in the same manner as those of $N_{2 n} / D_{2 n}$. Hence its decomposition into partial fractions gives such a sum as

$$
\sum \frac{M_{i}}{z+z_{i}}
$$$$
(z=1 / x)
$$

$M_{i}$ being positive as in (19). Now this gives rise to a series satisfying the condition $A_{n}>0 . \dagger \quad$ The reciprocal of the series (36) has therefore precisely the

*Annals of Mathematics, July, 1903.

† Stinltues, loc. cit., $§ 8$. 
same character as the series itself. Hence $2 p-2$ roots of the denominator of any approximant in the $(2 p-1)$ th diagonal file below the center of the table will be imaginary, while the roots of the numerator alternate on each side of the origin with the real roots of the denominator. This gives

Theorem X.-If $A_{n}>0$ and $q-p=2 n-1$, the number of imaginary roots of $V_{p q}$ will be $2 n-2$ and its real roots also will alternate with the roots of $U_{p q}$ on each side of the origin. If $p-q=2 n-1$, the polynomials $U_{p q}, V_{p q}$ are to be interchanged.

Wesleyan University, MidDletown, Conn. 